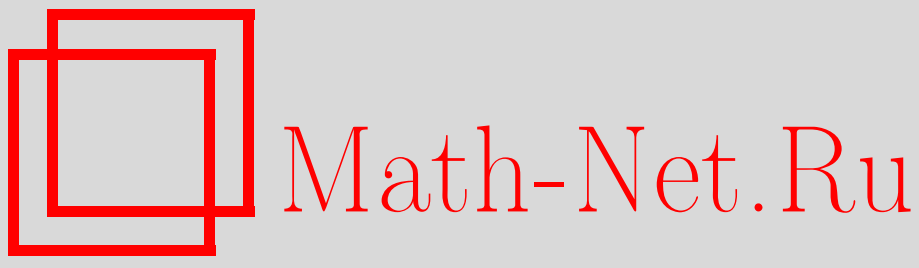

Д. Г. Маркушевич, А. С. Тихомиров, Симплектическая структура на пространстве модулей пучков на четырехмерной кубике, Изв. РАН. Сер. матем., 2003, том 67, выпуск 1, 131-158

DOI: https://doi.org/10.4213/im421

Использование Общероссийского математического портала Math-Net.Ru подразумевает, что вы прочитали и согласны с пользовательским соглашением

http://www . mathnet.ru/rus/agreement

Параметры загрузки:

IP : 3.80 .181 .102

26 апреля 2023 г., 14:28:28 
УДК 517.2

\author{
Д. Г. Маркушевич, А.С. Тихомиров
}

\title{
Симплектическая структура на пространстве модулей пучков на четырехмерной кубике
}

\begin{abstract}
Построено 10-мерное симплектическое пространство модулей пучков кручения на четырехмерной кубике в 5-мерном проективном пространстве. Оно параметризует стабильные векторные расслоения ранга 2 на гиперплоских сечениях четырехмерной кубики в 5-мерном проективном пространстве, получаемые конструкцией Серра из нормальных эллиптических квинтик. Естественная проекция построенного пространства модулей в двойственное 5-мерное проективное пространство является лагранжевым расслоением. Симплектическая структура оказывается тесно связанной (возможно, совпадающей) со спариванием Ионеды на пространстве модулей.

Библиография: 17 наименований.
\end{abstract}

\section{Введение}

В работе [12] Мукаи построил пример симплектического пространства модулей пучков кручения на К3-поверхности $S$, носители которых описывают полную линейную систему на $S$. Это пространство модулей идентично компактифицированному относительному якобиану линейной системы, и его слои, т. е. якобианы индивидуальных кривых, являются лагранжевыми подмногообразиями симплектической структуры.

Авторы настоящей статьи занимались поиском аналогичной ситуации с относительным промежуточным якобианом семейства многообразий высшей размерности вместо обычного якобиана семейства кривых. Поэтому соответствующие пучки кручения должны были бы иметь носители размерности по меньшей мере 3 , а их ограничения на носители имели бы ранг больше 1. Нами найдена 10-мерная компонента пространства модулей стабильных пучков с ожидаемыми свойствами, носители которых являются гиперплоскими сечениями 4-мерной гиперкубики в $\mathbb{P}^{5}$. Это новый пример пространства модулей пучков, обладающего симплектической структурой; известные ранее примеры таких пространств параметризуют пучки на многообразиях с голоморфными 2-формами (см. [12] для случая К3- либо абелевых поверхностей, [9] для гиперкэлеровых многообразий, [15] для поверхностей с $\left.p_{g}>0\right)$, и симплектические структуры на них по существу происходят из 2-формы на базе. В нашем примере база отстоит очень далеко от многообразий, обладающих голоморфной 2-формой: она является многообразием Фано.

Работа выполнена при частичной поддержке фонда INTAS-OPEN-2000-269.

(C) Д.Г. МАРкушевич, А.С. Тихомиров, 2003 
Слой пространства модулей, параметризуюший пучки с фиксированным носителем (3-мерной кубикой), был построен в предыдушей статье авторов [11]. В ней исследовалось отображение Абеля-Якоби семейства эллиптических кривых степени 5 (квинтик) на общей 3-мерной кубике. Нами доказано, что это отображение пропускается через отображение в 5 -мерную компоненту пространства модулей $M_{X}$ стабильных векторных расслоений $\mathscr{E}$ на $X$ с числами Черна $c_{1}=0, c_{2}=2$, общая точка которой представляет векторное расслоение, полученное конструкцией Серра из эллиптической квинтики $C \subset X$. Эллиптические квинтики, отображаюшиеся в точку пространства модулей, варьируются в 5 -мерном проективном пространстве внутри схемы Гильберта $\mathrm{Hilb}_{X}^{5 n}$, и индуцированное отображение $\Psi$ из компоненты пространства модулей в промежуточный якобиан $J(X)$ этально на открытом множестве, представляющем гладкие линейно нормальные эллиптические квинтики. Это отображение однозначно определяется выбором квинтики отсчета в $X$. В действительности эллиптические квинтики представляют класс $c_{2}(\mathscr{E}(1))$, поэтому можно интерпретировать $\Psi$ как вычисление класса $c_{2}$ со значениями в алгебраических циклах по модулю рациональной эквивалентности, взятого в композиции с отображением Абеля-Якоби на циклах.

В настоящей статье мы релятивизируем эту конструкцию над семейством неособых гиперплоских сечений четырехмерной кубики $Y$. Это дает 10-мерную компоненту пространства модулей $\mathfrak{M}_{Y}$ пучков кручения на $Y$ с носителями на гиперплоских сечениях $X=H \cap Y$, ограничения которых на $X$ являются векторными расслоениями ранга два из $M_{X}$. Мы показываем, что спаривание Ионеды индуцирует квазисимплектическую структуру на $\mathfrak{M}_{Y}$, т. е. невырожденную голоморфную 2-форму, которая необязательно замкнута, и что естественная проекция $p: \mathfrak{M} \rightarrow \check{\mathbb{P}}^{5}$ является лагранжевым расслоением, где $\check{\mathbb{P}}^{5}$ - база семейства гиперплоскостей в $\mathbb{P}^{5}$. Это означает, что слои $M_{X}$, параметризуюшие пучки с фиксированньм носителем $X$, являются лагранжевыми подмногообразиями в $\mathfrak{M}$.

Мы исследуем также связь квазисимплектической структуры Ионеды с симплектической структурой, построенной Донаги и Маркманом [5, пример 8.22] на многообразии $\mathbf{J}_{Y}$. Это многообразие обладает аналогичным свойством: слои $J(Y \cap H)$ являются лагранжевыми. Вообще говоря, не существует отображения между двумя 10-мерными многообразиями $\mathfrak{M}_{Y}$ и $\mathbf{J}_{Y}$, и нельзя сказать, что одна квазисимплектическая структура индуцирована другой. Тем не менее, отображение из $\mathfrak{M}_{Y}$ в $\mathbf{J}_{Y}$ существует локально над $\check{\mathbb{P}}^{5}$ в классической (либо этальной) топологии, и мы доказываем, что эти локальные отображения совместимы с симплектическими структурами с точностью до добавления 2-формы, которая является локальным сечением расслоения $p^{*} \Omega_{\mathbb{\mathbb { P } 5}}^{2}$.

Согласно [8] степень вышеуказанного отображения $\Psi$ равна 1 , поэтому $M_{X}$ можно отождествить с открытым подмножеством в $J^{2}(X)$, а $\mathfrak{M}_{Y}$ - с открытым подмножеством некоторого торсора над относительным промежуточным якобианом $\mathbf{J}_{Y}$ семейства неособых гиперплоских сечений $Y$.

$\mathrm{K}$ сожалению, не наблюдается какого-либо разумного подхода к доказательству замкнутости квазисимплектической структуры Ионеды. Заметим, что ни Тюрин, ни Мукаи не доказывали замкнутости их 2-форм. В итоге оказалось, что эти 2-формы замкнуты [13], [3], [7], но метод доказательства в указанных работах по су- 
шеству сводится к вопросу о замкнутости представителей класса Атьи когерентного пучка. Наша квазисимплектическая структура не содержит компонент класса Атьи, поэтому этот метод не работает. Тем не менее, мы доказываем сушествование симплектической структуры на $\mathfrak{M}_{Y}$, которая находится в том же соотношении со структурой Донаги-Маркмана: вышеуказанные локальные отображения из $\mathfrak{M}_{Y}$ в $\mathbf{J}_{Y}$ согласованы с симплектическими структурами с точностью до прибавления 2-формы, являющейся локальным сечением $p^{*} \Omega_{\mathbb{P}_{5}}^{2}$. Мы определяем ее фактически на торсоре, содержащем $\mathfrak{M}_{Y}$.

Опишем кратко содержание статьи по параграфам.

B $\S 1$ мыполучаем подготовительную информацию о когомологиях и Еxt-группах пучков из $\mathfrak{M}_{Y}$. Вычисления используют локально-относительную спектральную последовательность для Ext-пучков и проективную геометрию эллиптических квинтик.

В $\S 2$ мы доказьваем, что спаривание Ионеды $\operatorname{Ext}^{1}(\mathscr{E}, \mathscr{E}) \times \operatorname{Ext}^{1}(\mathscr{E}, \mathscr{E}) \stackrel{\text { Yoneda }}{\longrightarrow}$ $\operatorname{Ext}^{2}(\mathscr{E}, \mathscr{E}) \simeq \mathbb{C}$ для $\mathscr{E} \in \mathfrak{M}_{Y}$ является кососимметрическим и невырожденным отображением. Мы показываем, что комплексные прямые $\operatorname{Ext}^{2}(\mathscr{E}, \mathscr{E})$ для различных $\mathscr{E}$ склеиваются в тривиальное линейное расслоение над $\mathfrak{M}_{Y}$. Следовательно, спаривание Ионеды определяет настоящую 2-форму $\Lambda_{\mathfrak{M}_{Y}}$ на $\mathfrak{M}_{Y}$, дающую ожидаемую квазисимплектическую структуру.

В $\S 3$ мы описываем симплектическую структуру $\Lambda_{\mathbf{J}_{Y}}$ Донаги-Маркманана $\mathbf{J}_{Y}$. Она определяется симплектической структурой на 4-мерном многообразии $\Phi$ ано $Z$ многообразия $Y$, параметризующем прямые $l \subset Y$. Существование и единственность последней была доказана Бовилем и Донаги [2], но они не дали ее конструкцию.

В $\S 4$ дается явная конструкция симплектической структуры Бовиля-Донаги на $Z$. Идея построения воспроизводит доказательство теоремы о касательном расслоении для поверхности Фано трехмерной кубики. По существу, это доказательство есть вычисление дифференциала отображения Абеля-Якоби для семейства прямых посредством диаграммы Клеменса-Гриффитса-Велтерса (см. [17, § 2]) для тройки $l \subset X \subset \mathbb{P}^{4}$. Мы адаптируем его для тройки $l \subset Y \subset \mathbb{P}^{5}$, и это дает (после очевидной релятивизации) тензорное поле $\wedge_{Z}: \mathscr{T} Z \stackrel{\sim}{\rightarrow} \Omega_{Z}$. Принцип Бохнера гласит, что глобальные голоморфные тензорные поля на $Z$ порождены формой Бовиля-Донаги, поэтому форма $\wedge_{Z}$ должна быть ей пропорциональна.

В $\S 5$ мы сравниваем квазисимплектические структуры на $\mathfrak{M}_{Y}$ и $\mathbf{J}_{Y}$. Эти два многообразия расслоены над одной и той же базой $U \subset \check{\mathbb{P}}^{5}$, параметризующей неособые гиперплоские сечения $Y$, имеют лагранжевы слои, и эти слои связаны этальным отображением из [11]. Таким образом, можно поточечно отождествить касательные пространства двух соответствуюших слоев, равно как и их нормальные пространства, которые отождествляются с касательным пространством к $U$. Первое наблюдение состоит в том, что две квазисимплектические структуры определяют одинаковое спаривание между касательным и нормальным пространством к слою. Это дает поточечное отождествление двух квазисимплектических структур. Далее мы рассматриваем локальное (в классической топологии) послойное отображение $\Psi_{\mathscr{U}}: \mathfrak{M}_{Y} \rightarrow \mathbf{J}_{Y}$, которое является отображением Абеля-Якоби, определяемым семейством эллиптических квинтик отсчета в гиперплоских сече- 
ниях $Y$ над маленьким диском $\mathscr{U} \subset U$. Мы показываем, что $\Lambda_{\mathfrak{M}_{Y}}, \Lambda_{\mathbf{J}_{Y}}$ отличаются на сечение из $p^{*} \Omega_{\mathscr{U}}^{2}$ и что квазисимплектическую структуру Ионеды можно заменить на симплектическую структуру, связанную тем же отношением с $\Lambda_{\mathbf{J}_{Y}}$. В заключение мы обсуждаем продолжения на большее открытое подмножество $\bar{U}$, параметризуюшее гиперплоские сечения с не более чем одной невырожденной квадратичной особой точкой.

Первый автор с удовольствием выражает благодарность за гостеприимство Международному центру теоретической физики, г. Триест, и Математическому институту им. М. Планка, г. Бонн, где им была выполнена часть работы над статьей.

\section{$\S 1$. Вычисление Ext-групп}

Пусть $Y \subset \mathbb{P}^{5}$ - неособая четырехмерная кубика и $X=Y \cap \mathbb{P}^{4}$ - ее общее гиперплоское сечение. Пусть $\mathscr{E}$ - векторное расслоение, полученное из проективно нормальной эллиптической кривой пятой степени $C \subset X \subset \mathbb{P}^{4}$ посредством конструкции Серра:

$$
0 \rightarrow \mathscr{O}_{X} \rightarrow \mathscr{E}(1) \rightarrow \mathscr{I}_{C}(2) \rightarrow 0
$$

где $\mathscr{I}_{C}=\mathscr{I}_{C, X}$ - пучок идеалов $C$ в $X$. Поскольку класс $C$ по модулю алгебраической эквивалентности равен $5 l$, где $l$ - класс прямой, последовательность (1.1) влечет $c_{1}(\mathscr{E})=0, c_{2}(\mathscr{E})=2 l$. Более того, $\operatorname{det} \mathscr{E}$ тривиален, и, следовательно, $\mathscr{E}$ самодвойственно как векторное расслоение (т. е. $\mathscr{E}^{*} \simeq \mathscr{E}$ ). Дальнейшие детали этой конструкции см. в $[11, \S 2]$.

Здесь мы рассматриваем $\mathscr{E}$ как пучок кручения на $Y$ с носителем в $X$.

Лемма 1.1. Имеем

$$
\mathscr{E}_{\mathscr{O}_{Y}}^{q}(\mathscr{E}, \mathscr{E})= \begin{cases}\mathscr{E} \otimes \mathscr{E}, & \text { если } q=0, \\ \mathscr{E} \otimes \mathscr{E}(1), & \text { если } q=1, \\ 0 & \text { в противном случае. }\end{cases}
$$

ДоКАЗАТЕЛЬСТвО. Выберем локальную тривиализацию $\mathscr{E}:$

$$
\left.\left.\mathscr{E}\right|_{U} \simeq\left(\mathscr{O}_{X} \oplus \mathscr{O}_{X}\right)\right|_{U}
$$

Это сводит проблему к вычислению пучка $\mathscr{E} x t_{\mathscr{O}_{Y}}^{q}\left(\mathscr{O}_{X}, \mathscr{O}_{X}\right)$. Используя резольвенту

$$
0 \rightarrow \mathscr{O}_{Y}(-1) \rightarrow \mathscr{O}_{Y} \rightarrow \mathscr{O}_{X} \rightarrow 0,
$$

приходим к заключению, что $\mathscr{E} x t_{\mathscr{O}_{Y}}^{q}\left(\mathscr{O}_{X}, \mathscr{O}_{X}\right)=\mathscr{O}_{X}$ для $q=0, \mathscr{N}_{X / Y} \simeq \mathscr{O}_{X}(1)$ для $q=1$ и $\mathscr{N}_{X / Y}=0$ для $q \geqslant 2$. Принимая во внимание функториальный характер последнего изоморфизма по отношению к выбору базисов в $\mathscr{O}_{X}$, мы получаем искомые формулы с пучком $\mathscr{E}^{*} \otimes \mathscr{E}$ вместо $\mathscr{E} \otimes \mathscr{E}$, но эти пучки изоморфны в силу самодвойственности $\mathscr{E}$. 
ЛЕмма 1.2. Верны следующие утвержсдения:

(i) $h^{p}(\mathscr{E} \otimes \mathscr{E})=\operatorname{dim} \operatorname{Ext}_{X}^{p}(\mathscr{E} \otimes \mathscr{E})= \begin{cases}1, & \text { если } q=0, \\ 5, & \text { если } q=1, \\ 0 & \text { в противном случае; }\end{cases}$

(ii) $h^{0}(\mathscr{E}(1))=6 u h^{i}(\mathscr{E}(1))=0 \partial$ дя $i>0$;

(iii) $h^{0}\left(\mathscr{I}_{C}(k)\right)=\left(\begin{array}{c}4+k \\ 4\end{array}\right)-\left(\begin{array}{c}1+k \\ 4\end{array}\right)-5 k$ для $k \geqslant 1 u h^{i}\left(\mathscr{I}_{C}(k)\right)=0$, если $i>0$;

(iv) $h^{i}(\mathscr{E}(j))=0$ для всех $i \in \mathbb{Z}, \quad j=0,-1,-2$.

ДокАЗАТЕЛЬСтво. Утверждения (i), (ii) следуют из $[11$, лемма 2.1, c); лемма $2.7, \mathrm{~b})]$. Утверждение (iii) проверено для $k \geqslant 2$ в доказательстве леммы 2.1 из [11] и очевидным образом продолжается на случай $k=1$ рассмотрением точной тройки

$$
0 \rightarrow \mathscr{I}_{C}(k) \rightarrow \mathscr{O}_{X}(k) \rightarrow \mathscr{O}_{C}(k) \rightarrow 0
$$

с $k=1 ; C$ не содержится в гиперплоскости в силу проективной нормальности, поэтому $h^{0}\left(\mathscr{I}_{C}(1)\right)=0$. Так как $h^{i}\left(\mathscr{O}_{X}(1)\right)=h^{i}\left(\mathscr{O}_{C}(1)\right)$ равно 5 для $i=0$ и равно 0 для всех $i>0$, мы получаем искомое утверждение.

Утверждение (iv) для $j=1$ есть не что иное, как [11, лемма 2.7, a)]. По двойственности Серра (на $X$ ) остается проверить (iv) лишь для $j=0$. Это следует из (iii) с $k=1$, точной последовательности (1.1), подкрученной на $\mathscr{O}_{X}(-1)$, и равенств $H^{i}\left(\mathscr{O}_{X}(-1)\right)=0$ для $i>0$.

СлеДСТВиЕ 1.3. Локально-глобальная спектральная последовательность

$$
E_{2}^{p q}=H^{p}\left(Y, \mathscr{E} x t_{\mathscr{O}_{Y}}^{q}(\mathscr{E}, \mathscr{E})\right) \Longrightarrow \operatorname{Ext}_{Y}^{p+q}(\mathscr{E}, \mathscr{E})
$$

вирождается во втором илене: $E_{2}^{p q}=E_{\infty}^{p q}$. Следовательно, $\operatorname{Ext}_{Y}^{0}(\mathscr{E}, \mathscr{E})=\mathbb{C}$, m.е. $\mathscr{E}$ прост, $\operatorname{Ext}_{Y}^{1}(\mathscr{E}, \mathscr{E})$ включается в точную последовательность

$$
0 \rightarrow H^{1}(\mathscr{E} \otimes \mathscr{E}) \rightarrow \operatorname{Ext}_{Y}^{1}(\mathscr{E}, \mathscr{E}) \rightarrow H^{0}(\mathscr{E} \otimes \mathscr{E}(1)) \rightarrow 0
$$

$u \operatorname{Ext}_{Y}^{n}(\mathscr{E}, \mathscr{E})=H^{n-1}(\mathscr{E} \otimes \mathscr{E}(1)) \partial \Omega я n \geqslant 2$.

ДоКАЗАТЕЛЬСТВО. Это немедленно следует из леммы 1.1 и утверждения (i) леммы 1.2 .

ЛЕмма 1.4. Выполняются следующие утверждения:

(i) $H^{i}(\mathscr{E} \otimes \mathscr{E})=H^{i}\left(\mathscr{E} \otimes \mathscr{I}_{C}(1)\right) u H^{i}(\mathscr{E} \otimes \mathscr{E}(1))=H^{i}\left(\mathscr{E} \otimes \mathscr{I}_{C}(2)\right)$ для всех $i \in \mathbb{Z}$;

(ii) $H^{i}(\mathscr{E} \otimes \mathscr{E}(1))=0 \partial \Omega \mathscr{A} i=2,3 u \chi(\mathscr{E} \otimes \mathscr{E}(1))=h^{0}(\mathscr{E} \otimes \mathscr{E}(1))-h^{1}(\mathscr{E} \otimes$ $\mathscr{E}(1))=4$. 
ДокАЗАТЕЛЬСтво. Первый изоморфизм доказан в [11]. Для доказательства второго изоморфизма рассмотрим точные последовательности

$$
\begin{aligned}
& 0 \rightarrow \mathscr{E} \rightarrow \mathscr{E} \otimes \mathscr{E}(1) \rightarrow \mathscr{I}_{C} \otimes \mathscr{E}(2) \rightarrow 0, \\
& 0 \rightarrow \mathscr{I}_{C} \otimes \mathscr{E}(2) \rightarrow \mathscr{E}(2) \rightarrow \mathscr{E}(2) \otimes \mathscr{O}_{C} \rightarrow 0,
\end{aligned}
$$

получаемые тензорным умножением $(1.1),(1.2)$ на $\mathscr{E}$.

Утверждение (i) следует из (1.3) и леммы 1.2, (iv).

Утверждения (ii) и (iv) леммы 1.2 дают достаточно много значений эйлеровой характеристики $\chi(\mathscr{E}(k))$, которая есть кубический полином от $k$ по теореме Римана-Роха-Хирцебруха, и мы получаем, что $\chi(\mathscr{E}(k))=k(k+1)(k+2)$. Таким образом, $\chi(\mathscr{E}(2))=24$. Имеем также $\operatorname{deg} \mathscr{E}(2) \otimes \mathscr{O}_{C}=20$, следовательно, $\chi\left(\mathscr{E}(2) \otimes \mathscr{O}_{C}\right)=20$, и это позволяет определить оставшиеся числа Эйлера из $(1.3),(1.4)$, доказьвая тем самым равенство $\chi(\mathscr{E} \otimes \mathscr{E}(1))=4$.

Теперь снова используем (1.4), чтобы показать, что $H^{i}\left(\mathscr{E} \otimes \mathscr{I}_{C}(2)\right)=0$ для $i=2,3$. Сначала покажем, что $H^{1}\left(\mathscr{E}(2) \otimes \mathscr{O}_{C}\right)=0$. В силу конструкции (1.1) $\left.\mathscr{E}(1)\right|_{C} \simeq \mathscr{N}_{C / X}$. Следовательно,

$$
H^{1}\left(\mathscr{E}(2) \otimes \mathscr{O}_{C}\right)=H^{1}\left(\mathscr{N}_{C / X}(1)\right)=H^{0}\left(\mathscr{N}_{C / X}^{*}(-1)\right)^{*}=H^{0}\left(\mathscr{N}_{C / X}(-3)\right)^{*}
$$

(мы использовали тот факт, что $\mathscr{N}_{C / X}(-1)$ самодвойственно).

Рассмотрим естественное включение $\mathscr{N}_{C / X}(-2) \subset \mathscr{N}_{C / \mathbb{P}^{4}}(-2)$ и в силу $[6$, предложение V.2.1] имеем $H^{1}\left(\mathscr{N}_{C / \mathbb{P}^{4}}^{*}(2) \otimes \mathscr{M}\right)=0$ для любого обратимого пучка $\mathscr{M}$ степени 0. По двойственности Серра $H^{0}\left(\mathscr{N}_{C / \mathbb{P}^{4}}(-2)\right)=0$. Значит, $H^{0}\left(\mathscr{N}_{C / X}(-2)\right)=$ 0 и, тем более, $H^{0}\left(\mathscr{N}_{C / X}(-3)\right)=0$. Следовательно, $H^{1}\left(\mathscr{N}_{C / X}(1)\right)=0$.

Далее, $H^{i}(\mathscr{E}(2))=0$ для $i>0$ в силу того же самого рассуждения, которое применялось в доказательстве утверждения (ii) леммы 1.2. Теперь из (1.4) получаем требуемое утверждение.

Лемма 1.5. Имеют место равенства $h^{0}\left(\mathscr{I}_{C}^{2}(3)\right)=0, h^{1}\left(\mathscr{E}(2) \otimes \mathscr{I}_{C}\right)=1$ $u$, тем самым,$h^{0}\left(\mathscr{E}(2) \otimes \mathscr{I}_{C}\right)=5$.

ДокАЗАТЕЛЬСТво. Как и в доказательстве леммы VI.3.1 из [6], мы имеем точную последовательность

$$
0 \rightarrow \mathscr{F} \rightarrow H^{0}\left(\mathscr{I}_{C / \mathbb{P}^{4}}(2)\right) \otimes \mathscr{O}_{C} \stackrel{\alpha}{\rightarrow} \mathscr{N}_{C / \mathbb{P}^{4}}^{*}(2) \rightarrow 0,
$$

где $\mathscr{F}=\operatorname{ker} \alpha-$ векторное расслоение ранга $2 \mathrm{c} \operatorname{det} \mathscr{F}=\mathscr{O}_{C}(-1)$ и $h^{0}(\mathscr{F})=0$. Оно является либо прямой суммой двух линейных расслоений $\mathscr{F}=\mathscr{M}_{1} \oplus \mathscr{M}_{2}$, $\operatorname{deg} M_{i} \geqslant-5$, либо включается в нерасщепляющуюся точную последовательность

$$
0 \rightarrow \mathscr{O}_{C}(-3 o) \otimes \mathscr{M} \rightarrow \mathscr{F} \rightarrow \mathscr{O}_{C}(-2 o) \otimes \mathscr{M} \rightarrow 0,
$$

где $\mathscr{M}$ - тэта-характеристика на $C$, а $o$ - точка в $C$ такая, что $\mathscr{O}_{C}(1) \simeq \mathscr{O}_{C}(5 o)$. Это влечет $h^{1}(\mathscr{F}(1))=0$. Согласно (1.5) отображение

$$
H^{0}\left(\mathscr{I}_{C / \mathbb{P}^{4}}(2)\right) \otimes H^{0}\left(\mathscr{O}_{C}(1)\right) \rightarrow H^{0}\left(\mathscr{N}_{C / \mathbb{P}^{4}}^{*}(3)\right)
$$


сюръективно. Это отображение пропускается, очевидно, через $H^{0}\left(\mathscr{I}_{C / \mathbb{P}^{4}}(3)\right)$, следовательно, отображение

$$
H^{0}\left(\mathscr{I}_{C / \mathbb{P}^{4}}(3)\right) \rightarrow H^{0}\left(\mathscr{N}_{C / \mathbb{P}^{4}}^{*}(3)\right)
$$

также сюръективно. По теореме Римана-Роха $h^{0}(\mathscr{F}(1))=5$, и в силу $(1.5)$ $h^{0}\left(\mathcal{N}_{C / \mathbb{P}^{4}}^{*}(3)\right)=25-5=20$ и $h^{1}\left(\mathscr{N}_{C / \mathbb{P}^{4}}^{*}(3)\right)=0$. Согласно $[11$, лемма 4.2], $h^{0}\left(\mathscr{I}_{C / \mathbb{P}^{4}}(3)\right)=20$, следовательно, точная последовательность

$$
0 \rightarrow \mathscr{I}_{C / \mathbb{P}^{4}}^{2}(3) \rightarrow \mathscr{I}_{C / \mathbb{P}^{4}}(3) \rightarrow \mathscr{N}_{C / \mathbb{P}^{4}}^{*}(3) \rightarrow 0
$$

дает равенство $h^{0}\left(\mathscr{I}_{C / \mathbb{P}^{4}}^{2}(3)\right)=0$. В силу проективной нормальности кривой $C$ имеем $h^{1}\left(\mathscr{I}_{C / \mathbb{P}^{4}}(3)\right)=0$, а значит, $h^{1}\left(\mathscr{I}_{C / \mathbb{P}^{4}}^{2}(3)\right)=0$.

Рассмотрим следующую коммутативную диаграмму с точными строками и столбцами:

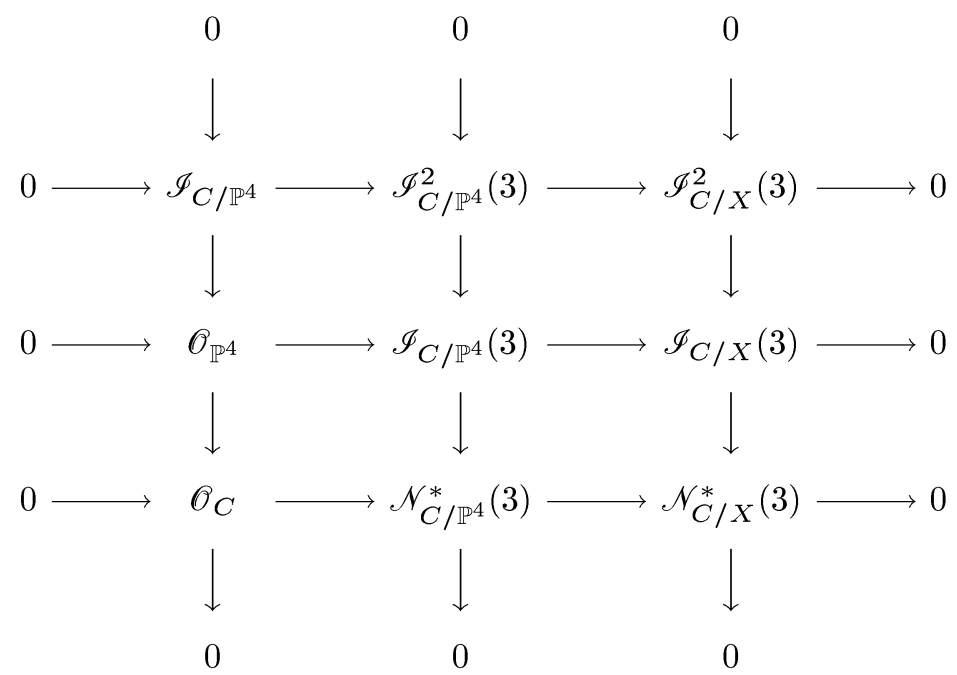

Из левого столбца получаем равенство $h^{1}\left(\mathscr{I}_{C / \mathbb{P}^{4}}\right)=0$, из первой строки находим $h^{0}\left(\mathscr{I}_{C / X}^{2}(3)\right)=h^{1}\left(\mathscr{I}_{C / \mathbb{P}^{4}}\right)=0$. Из нижней строки имеем $h^{0}\left(\mathscr{N}_{C / X}^{*}(3)\right)=20$, $h^{1}\left(\mathscr{N}_{C / X}^{*}(3)\right)=0$, а из средней строки $-h^{0}\left(\mathscr{I}_{C / X}(3)\right)=19, h^{1}\left(\mathscr{I}_{C / X}(3)\right)=0$, откуда следует, что $h^{1}\left(\mathscr{I}_{C / X}^{2}(3)\right)=1$. 
Теперь рассмотрим другую коммутативную диаграмму с точными тройками:

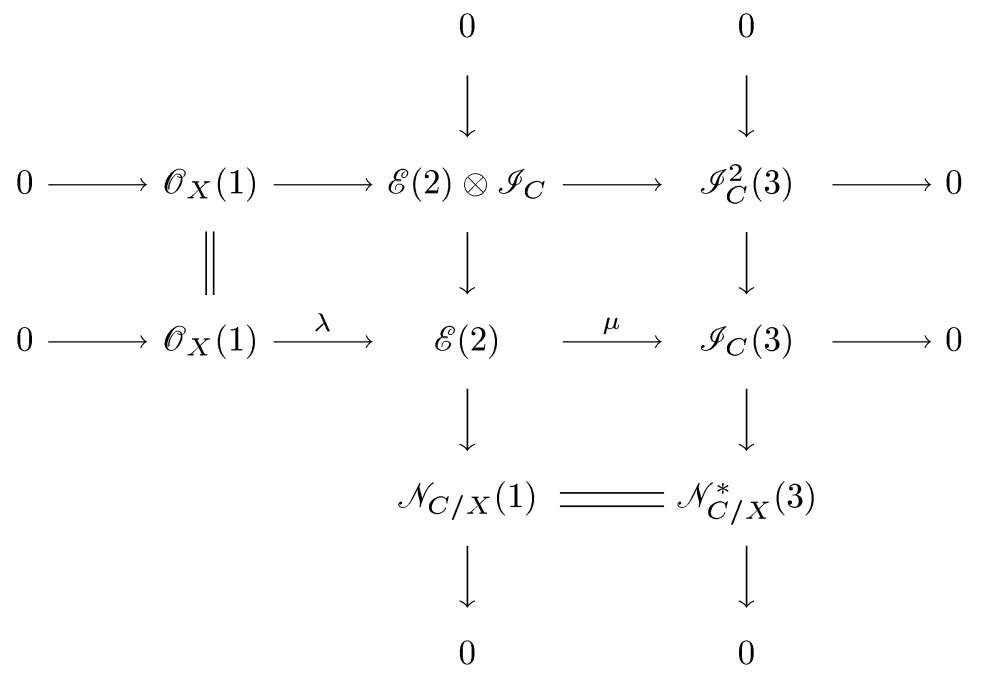

Из средней строки видно, что $\mu$ сюръективно на глобальных сечениях. Можно рассматривать элементы из $H^{0}\left(\mathscr{E}(2) \otimes \mathscr{I}_{C}\right)$ как сечения из $H^{0}(\mathscr{E}(2))$, обрашающиеся в нуль на $C$. Таким образом, сечение $s \in H^{0}(\mathscr{E}(2))$ лежит в $H^{0}\left(\mathscr{E}(2) \otimes \mathscr{I}_{C}\right)$ тогда и только тогда, когда $\mu(s) \in H^{0}\left(\mathscr{I}_{C / X}^{2}(3)\right)$. Следовательно,

$$
\begin{aligned}
H^{0}\left(\mathscr{E}(2) \otimes \mathscr{I}_{C}\right) & =\mu^{-1}\left(H^{0}\left(\mathscr{I}_{C / X}^{2}(3)\right)\right), \\
h^{0}\left(\mathscr{E}(2) \otimes \mathscr{I}_{C}\right) & =h^{0}\left(\mathscr{I}_{C / X}^{2}(3)\right)+\operatorname{dim} \operatorname{ker} \mu=5
\end{aligned}
$$

и $h^{1}\left(\mathscr{E}(2) \otimes \mathscr{I}_{C}\right)=1$ по лемме 1.4 , (ii). Лемма доказана.

СлеДСтвИЕ 1.6. Имеем $\operatorname{dim}_{\operatorname{Ext}_{Y}^{1}(\mathscr{E}, \mathscr{E})}=10, \quad \operatorname{dim}_{\operatorname{Ext}_{Y}^{2}(\mathscr{E}, \mathscr{E})}=1$, $\operatorname{Ext}_{Y}^{i}(\mathscr{E}, \mathscr{E})=0 \quad \partial \Omega \mathscr{S} i>2$.

ДокАЗАТЕЛЬСТвО. Эти равенства вытекают из следствия 1.3 и леммы 1.4.

\section{§2. Квазисимплектическая структура на $\mathfrak{M}_{Y}$}

Пусть $Y$ - неособая четырехмерная кубика. Обозначение $X$ будет использоваться для произвольного гиперплоского сечения кубики $Y$. Пусть $\mathscr{M}$ - объединение компонент пространства модулей простых пучков на $Y$, содержащего классы $[\mathscr{E}]$ пучков $\mathscr{E}$, определяемых тройками (1.1). Согласно [11] семейство проективно нормальных эллиптических квинтик на общей трехмерной кубике неприводимо, так что $\mathscr{M}$ неприводимо в случае, когда $Y$ - обшая четырехмерная кубика. Пусть $\mathfrak{M} \subset \mathscr{M}$ - подмножество классов пучков $\mathscr{E}$, определяемых расширениями (1.1) для всех проективно нормальных эллиптических квинтик $C$, лежащих на всех неособых гиперплоских сечениях $Y$.

ПРЕДЛОЖЕНИЕ 2.1. (i) $\mathfrak{M}$ есть открытое подмножсество в $\mathscr{M}$, содержащееся во множестве гладких точек пространства модулей простых пучков. 
(ii) Для всех $i, \quad p, \quad q$ әруппь $\operatorname{Ext}_{Y}^{i}(\mathscr{E}, \mathscr{E}), H^{p}\left(Y, \mathscr{E} x t_{\mathscr{O}_{Y}}^{q}(\mathscr{E}, \mathscr{E})\right)$ для различных $\mathscr{E}$ склеиваются (как векторные пространства) в векторные расслоения $\mathscr{T}^{i}$, соответственно, $\mathscr{T}^{p, q}$ на М. Другими словами, существуют такие векторные расслоения $\mathscr{T}^{i}, \mathscr{T}^{p, q}$ на $\mathfrak{M}$, что для любого $\mathscr{E}, \quad e=[\mathscr{E}] \in \mathfrak{M}$, имеют место канонические изоморфизмы $\mathscr{T}^{i} \otimes \mathbb{C}(e)=\operatorname{Ext}_{Y}^{i}(\mathscr{E}, \mathscr{E}), \mathscr{T}^{p, q} \otimes$ $\mathbb{C}($ e $)=H^{p}\left(Y, \mathscr{E} x t_{\mathscr{O}_{Y}}^{q}(\mathscr{E}, \mathscr{E})\right)$. Существует каноническое включение $\mathscr{T}^{i, 0} \subset \mathscr{T}^{i}$. Расслоение $\mathscr{T}^{1}$ канонически изоморфно касательному расслоению $\kappa \mathfrak{M}, u$ $\operatorname{rk} \mathscr{T}^{1}=\operatorname{dim} \mathfrak{M}=10, \operatorname{rk} \mathscr{T}^{2}=1$.

(iii) Спаривание Ионеды

$$
\operatorname{Ext}_{Y}^{i}(\mathscr{E}, \mathscr{E}) \times \operatorname{Ext}_{Y}^{j}(\mathscr{E}, \mathscr{E}) \rightarrow \operatorname{Ext}_{Y}^{i+j}(\mathscr{E}, \mathscr{E})
$$

определяет кососимметрическую билинейную форму

$$
\Lambda: \mathscr{T}^{1} \times \mathscr{T}^{1} \rightarrow \mathscr{T}^{2}
$$

и $\mathscr{T}^{1,0}$ изотропно по отношению $\kappa \Lambda$.

ДоказАтельство. (i) Для доказательства гладкости заметим, что $\operatorname{dim} \mathfrak{M} \leqslant$ $10=\operatorname{dim} \operatorname{Ext}_{Y}^{1}(\mathscr{E}, \mathscr{E})$, и равенство здесь имеет место тогда и только тогда, когда $\mathscr{M}$ неособо в $[\mathscr{E}]$. Но это равенство на самом деле выполнено всегда, потому что $\mathfrak{M}$ содержит явно 10-мерное семейство попарно неизоморфных пучков: мы имеем 5 параметров, определяющих носитель $X$ пучка, и пространство модулей $M_{X}$ пучков с фиксированным носителем $X$ неособо и имеет размерность 5 согласно [11]. Открытость $\mathfrak{M}$ в пространстве модулей пучков на $Y$ получается дословно так же, как и в $[11$, следствие 5.5], с использованием соответствующей релятивизации конструкции Серра.

Существование пучков $\mathscr{T}^{i}$ в (ii) и билинейной формы в (iii) следует из [12, предложение 2.2] и [14, теорема 1.21,(4)]. Доказательство сушествования $\mathscr{T}^{p, q}$ получается очевидной модификацией аргументов из [12]. Кососимметричность вытекает из гладкости: квадрат Ионеды $z$ о $z$ элемента $z \in \operatorname{Ext}_{Y}^{1}(\mathscr{E}, \mathscr{E})$ есть в точности отображение первого препятствия $\operatorname{Ext}_{Y}^{1}(\mathscr{E}, \mathscr{E}) \rightarrow \operatorname{Ext}_{Y}^{2}(\mathscr{E}, \mathscr{E})$, и оно является нулевым в силу гладкости $\mathscr{M}$ в $\mathscr{E}$.

По общим свойствам спектральных последовательностей композиции функторов с кольцевой структурой спаривание Ионеды индуцирует на биградуированном объекте $E_{2}^{p q}$ естественную кольцевую структуру

$$
H^{p}\left(Y, \mathscr{E} x t_{\mathscr{O}_{Y}}^{q}(\mathscr{E}, \mathscr{E})\right) \times H^{p^{\prime}}\left(Y, \mathscr{E} x t_{\mathscr{O}_{Y}}^{q^{\prime}}(\mathscr{E}, \mathscr{E})\right) \rightarrow H^{p+p^{\prime}}\left(Y, \mathscr{E} x t_{\mathscr{O}_{Y}}^{q+q^{\prime}}(\mathscr{E}, \mathscr{E})\right)
$$

Изотропность $H^{1}(\mathscr{E} \otimes \mathscr{E})$ вытекает из того факта, что $H^{2}(\mathscr{E} \otimes \mathscr{E})=0$ в силу леммы $1.2,(\mathrm{i})$.

Для того чтобы получить квазисимплектическую структуру, необходимо проверить, что $\Lambda$ невырождена и что $\mathscr{T}^{2}$ есть тривиальное линейное расслоение. 
Ввиду изотропности $H^{1}(\mathscr{E} \otimes \mathscr{E})$ невырожденность спаривания $\operatorname{Ext}_{Y}^{1}(\mathscr{E}, \mathscr{E}) \times$ $\operatorname{Ext}_{Y}^{1}(\mathscr{E}, \mathscr{E}) \rightarrow \operatorname{Ext}_{Y}^{2}(\mathscr{E}, \mathscr{E})$ равносильна невырожденности спаривания

$$
H^{1}(\mathscr{E} \otimes \mathscr{E}) \times H^{0}(\mathscr{E} \otimes \mathscr{E}(1)) \rightarrow H^{1}(\mathscr{E} \otimes \mathscr{E}(1))
$$

Теперь, используя изоморфизм $\wedge^{2} \mathscr{E} \simeq \mathscr{O}_{X}$, можно отождествить $H^{0}(\mathscr{E} \otimes \mathscr{E}(1))$ с $H^{0}\left(\wedge^{2} \mathscr{E}(1)\right)=H^{0}\left(\mathscr{O}_{X}(1)\right)$ посредством естественного отображения $\mathscr{E} \otimes \mathscr{E} \rightarrow \wedge^{2} \mathscr{E}$. Тот факт, что результируюшее отображение является изоморфизмом, следует из разложения

$$
H^{0}(\mathscr{E} \otimes \mathscr{E}(1))=H^{0}\left(\mathscr{I}_{C} \otimes \mathscr{E}(2)\right)=H^{0}\left(\mathscr{O}_{X}(1)\right)
$$

вытекающего из (1.3) и верхней строки в (1.7).

ЛЕмма 2.2. Спаривание (2.2) отождествляется посредством вышеуказанного изоморфизма с простым умножением әлементов из $H^{1}(\mathscr{E} \otimes \mathscr{E})$ на линейнье формы:

$$
H^{1}(\mathscr{E} \otimes \mathscr{E}) \times H^{0}\left(\mathscr{O}_{X}(1)\right) \rightarrow H^{1}(\mathscr{E} \otimes \mathscr{E}(1))
$$

ДокАЗАТЕЛЬСТво. Чтобы показать, что эти два спаривания совпадают, выразим их в локальном базисе в $\mathscr{E}$. Второе спаривание есть просто умножение коэффициентов:

$$
\left(\sum a_{i j} e_{i} \otimes e_{j}, l\right) \mapsto \sum l a_{i j} e_{i} \otimes e_{j}
$$

Выводим теперь локальную формулу для первого спаривания. Заметим, что $\mathscr{E} \otimes \mathscr{E}$ содержит тривиальное подрасслоение $\wedge^{2} \mathscr{E}$ в качестве прямого слагаемого. Согласно предыдущим рассмотрениям $H^{0}(\mathscr{E} \otimes \mathscr{E}(1))=H^{0}\left(\wedge^{2} \mathscr{E}(1)\right)$. Следовательно, каждое сечение из $H^{0}(\mathscr{E} \otimes \mathscr{E}(1))$ может быть локально записано как $l\left(e_{2} \otimes e_{1}-\right.$ $\left.e_{1} \otimes e_{2}\right)$. Задавая самодвойственность расслоения $\mathscr{E}$ как тривиализацию $\wedge^{2} \mathscr{E}$, можно считать, что $e_{2} \wedge e_{1}=e_{2} \otimes e_{1}-e_{1} \otimes e_{2}$ есть тривиализующее сечение, и тогда двойственньй базис для $\left(e_{j}\right)=\left(e_{1}, e_{2}\right)$ есть $\left((-1)^{\bar{j}} e_{\bar{j}}\right)=\left(e_{2},-e_{1}\right)$, где $\bar{j}=3-j$. Поэтому свертка средних членов

$$
\left(a_{1} \otimes a_{2}, b_{1} \otimes b_{2}\right) \mapsto \frac{a_{2} \wedge b_{1}}{e_{2} \wedge e_{1}} a_{1} \otimes b_{2},
$$

даюшая спаривание Ионеды, есть тождественное отображение $e_{i} \otimes e_{j} \mapsto e_{i} \otimes e_{j}$ на базисных сечениях. Отсюда вытекает, что спаривание Ионеды задается умножением коэффициентов $\left(a_{i j}, l\right) \mapsto l a_{i j}$. Лемма доказана.

ПРЕДЛОЖЕНИЕ 2.3. Спаривание (2.3) невырождено. 
ДОКАЗАТЕЛЬСТВО. Нам нужно проверить, что для любой линейной формы $l \in$ $H^{0}\left(\mathscr{O}_{X}(1)\right)$ отображение

$$
\cdot l: H^{1}(\mathscr{E} \otimes \mathscr{E}) \rightarrow H^{1}(\mathscr{E} \otimes \mathscr{E}(1))(\simeq \mathbb{C})
$$

сюръективно. По лемме 1.4 последнее отображение отождествляется с

$$
\cdot l: H^{1}\left(\mathscr{I}_{C} \otimes \mathscr{E}(1)\right) \rightarrow H^{1}\left(\mathscr{I}_{C} \otimes \mathscr{E}(2)\right) .
$$

Используя диаграмму (1.6), подкрученную на $\mathscr{O}(-1)$, и лемму 1.4, (i), можно продолжить последовательность отождествлений:

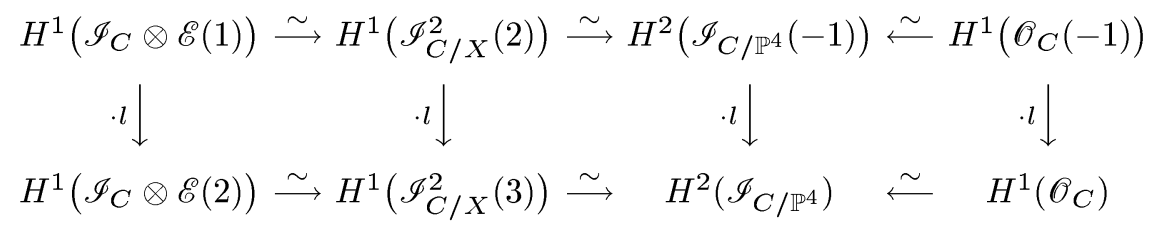

Здесь нуждаются в доказательстве лишь изоморфизмы, включаюшие $\mathscr{I}_{C / X}^{2}$. Те из них, которые содержат $H^{1}\left(\mathscr{I}_{C / X}^{2}(3)\right)$, установлены в доказательстве леммы 1.5 . Проверим теперь изоморфизмы, включаюшие $H^{1}\left(\mathscr{I}_{C / X}^{2}(2)\right)$. Для этого выпишем диаграмму (1.6), подкрученную на $\mathscr{O}(-1)$ :

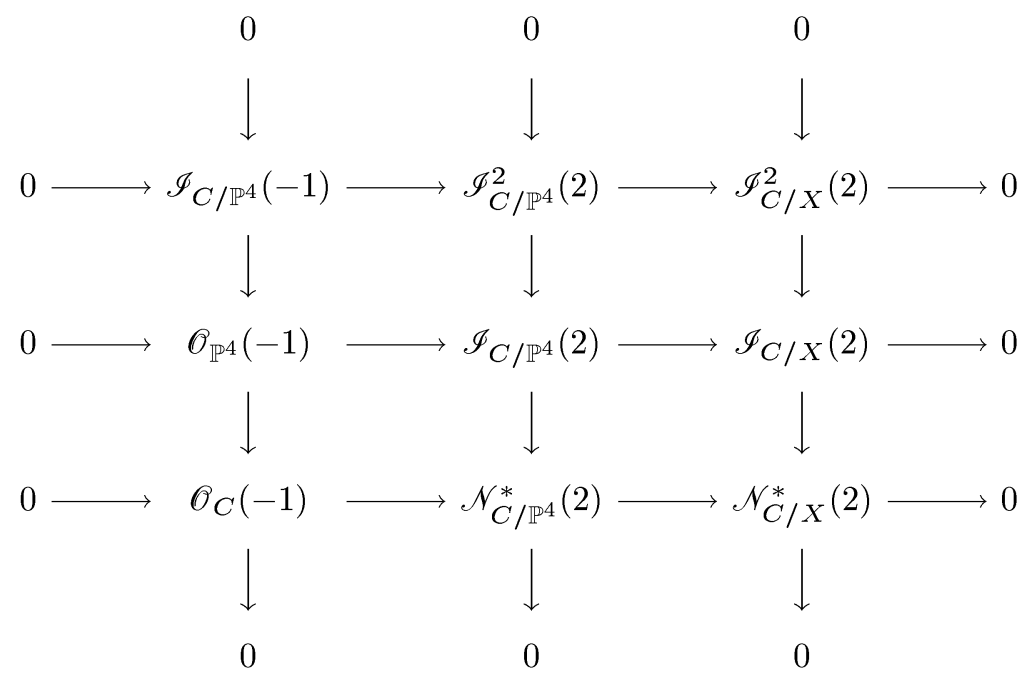

Из левого столбца получаем для вектора $\left(h^{i}\left(\mathscr{I}_{C / \mathbb{P}^{4}}(-1)\right)\right)$ значения $(0,0,5$, $0, \ldots)$. Аналогично, из левого столбца диаграммы (1.6) получаем $\left(h^{i}\left(\mathscr{I}_{C / \mathbb{P}^{4}}(2)\right)\right)=$ $(5,0,0, \ldots)$. Теперь из средней строки в $(2.5)$ имеем $\left(h^{i}\left(\mathscr{I}_{C / X}(2)\right)\right)=(5,0,0, \ldots)$. Далее, $\mathscr{N}_{C / X}^{*}(2) \simeq \mathscr{N}_{C / X}$, и по $\left[11\right.$, лемма 5.1, е)] $\left(h^{i}\left(\mathscr{N}_{C / X}^{*}(2)\right)\right)=(10,0,0, \ldots)$. Мы доказали в предыдушем параграффе, что $h^{0}\left(\mathscr{I}_{C / X}^{2}(3)\right)=0$, а потому и подавно $h^{0}\left(\mathscr{I}_{C / X}^{2}(2)\right)=0$. Следовательно, из первого столбца в $(2.5)$ получаем, что $\left(h^{i}\left(\mathscr{I}_{C / X}^{2}(2)\right)\right)=(0,5,0, \ldots)$. 
Доказано, что $h^{2}\left(\mathscr{I}_{C / \mathbb{P}^{4}}(-1)\right)=h^{1}\left(\mathscr{I}_{C / X}^{2}(2)\right)=5$. Теперь проверим, что связывающий гомоморфизм $H^{1} \rightarrow H^{2}$ в первой строке в действительности есть изоморфизм. Рассмотрим второй столбец в (2.5). Согласно [6, предложение V.2.1] имеем $\left(h^{i}\left(\mathscr{N}_{C / \mathbb{P}^{4}}^{*}(2)\right)\right)=(5,0,0, \ldots)$. Мы видели в предыдущем параграффе, что $h^{0}\left(\mathscr{I}_{C / \mathbb{P}^{4}}^{2}(3)\right)=0$, и поэтому тем более $h^{0}\left(\mathscr{I}_{C / \mathbb{P}^{4}}^{2}(2)\right)=0$. Следовательно, $\left(h^{i}\left(\mathscr{J}_{C / \mathbb{P}^{4}}^{2}(2)\right)\right)=(0,0,0, \ldots)$. Поэтому все связывающие гомоморфизмы в первой строке являются изоморфизмами.

Теперь, возврашаясь к диаграмме (2.4), мы видим, что вертикальное отображение в правой части очевидным образом сюръективно. Предложение доказано.

Теорема 2.4. Линейное расслоение $\mathscr{T}^{2}$ на $\mathfrak{M}$ тривиально, а следовательно, кососимметрическое спаривание $\Lambda$, определенное в (2.1), есть корректно определенная 2-форма на $\mathfrak{M}$ :

$$
\Lambda: \mathscr{T} \mathfrak{M} \stackrel{\sim}{\rightarrow} \Omega_{\mathfrak{M}}^{1}, \quad \Lambda \in H^{0}\left(\Omega_{\mathfrak{M}}^{2}\right)
$$

Слои естественного отображения $p: \mathfrak{M} \rightarrow \check{\mathbb{P}}^{5}:[\mathscr{E}] \mapsto\langle\operatorname{Supp}(\mathscr{E})\rangle$ являются лагранжевыми подмногообразиями по отношению $\kappa \Lambda$.

ДоКАЗАТЕЛЬСТВО. По предложению 2.1 для каждой точки $e=[\mathscr{E}] \in \mathfrak{M}$ имеется канонический изоморфизм $\mathscr{T}^{2} \otimes \mathbb{C}(e) \simeq \operatorname{Ext}_{Y}^{2}(\mathscr{E}, \mathscr{E})$. Достаточно показать, что имеется канонический изоморфизм

$$
\alpha: \operatorname{Ext}_{Y}^{2}(\mathscr{E}, \mathscr{E}) \stackrel{\text { can }}{\simeq} \mathbb{C} .
$$

С этой целью зафиксируем уравнение $f_{Y}=0$ кубики $Y$ в $\mathbb{P}^{5}$ и возьмем произвольное сечение $h \in H^{0}\left(\mathscr{O}_{Y}(1)\right)$, обрашающееся в нуль на $X=\operatorname{Supp}(\mathscr{E}) ;$ это сечение $h$ определяет изоморфизм $\phi_{h}: \mathscr{O}_{X}(1) \stackrel{\sim}{\rightarrow} \mathscr{N}_{X / Y}$ такой, что подстановка $h \mapsto \lambda h$ индуцирует $\phi_{h} \mapsto \lambda^{-1} \phi_{h}$.

Далее, по лемме 1.1 имеет место канонический изоморфизм

$$
\mathscr{E} x t_{\mathscr{O}_{Y}}^{1}(\mathscr{E}, \mathscr{E}) \stackrel{\text { can }}{\rightarrow} \mathscr{E}^{\vee} \otimes \mathscr{E} \otimes \mathscr{N}_{X / Y}
$$

Возьмем общее сечение $0 \neq s \in H^{0}(\mathscr{E}(1))$ такое, что $C=(s)_{0}$ есть гладкая проективно нормальная эллиптическая квинтика. Согласно вычислениям, проведенным в $\S 1$,

$$
h^{i}\left(\operatorname{det} \mathscr{E} \otimes \mathscr{I}_{C, H}^{2}(3)\right)=h^{i}\left(\mathscr{I}_{C, H}^{2}(3)\right)=0, \quad i=1,2,
$$

где $H=\langle C\rangle=\langle X\rangle$ - линейная оболочка кривой $C$, т. е. кубики $X$ в $\mathbb{P}^{5}$. Это сечение $s$ определяет точную последовательность

$$
0 \rightarrow \mathscr{O}_{X} \stackrel{s}{\rightarrow} \mathscr{E}(1) \stackrel{\cdot \wedge s}{\rightarrow} \mathscr{I}_{C, X}(2) \otimes \operatorname{det} \mathscr{E} \rightarrow 0
$$


Подкручивая ее на $\mathscr{E}^{\vee}$ и используя канонический изоморфизм $\mathscr{E} \vee \otimes \operatorname{det} \stackrel{\mathscr{c}}{\mathscr{c}} \stackrel{\sim}{\rightarrow} \mathscr{E}$, получаем точную тройку $0 \rightarrow \mathscr{E}^{\vee} \rightarrow \mathscr{E}^{\vee} \otimes \mathscr{E}(1) \stackrel{\varepsilon_{s}}{\rightarrow} \mathscr{E} \otimes \mathscr{I}_{C / X}(2) \rightarrow 0$. Таким образом, мы имеем изоморфизм композищии (Cor .1.3 - изоморфизм, имеющий место согласно следствию 1.3)

$$
\begin{aligned}
& \gamma_{1}: \operatorname{Ext}_{Y}^{2}(\mathscr{E}, \mathscr{E}) \underset{\mathrm{Cor} .1 .3}{\stackrel{\operatorname{can}}{\simeq}} H^{1}\left(\mathscr{E} x t_{\mathscr{O}_{Y}}^{1}(\mathscr{E}, \mathscr{E})\right) \stackrel{\text { can }}{\simeq} H^{1}\left(\mathscr{E} \vee \otimes \mathscr{E} \otimes \mathscr{N}_{X / Y}\right) \\
& \underset{\phi_{h}^{-1}}{\longrightarrow} H^{1}(\mathscr{E} \vee \otimes \mathscr{E}(1)) \stackrel{H^{1}\left(\varepsilon_{s}\right)}{\longrightarrow} H^{1}\left(\mathscr{E} \otimes \mathscr{I}_{C, X}(2)\right)
\end{aligned}
$$

который согласно сказанному вьше пропорционален ${ }^{1} h$ и $s$. Далее, аналогично (2.10) мы имеем морфизм $\mathscr{E} \otimes \mathscr{I}_{C, X}(2) \stackrel{\wedge s}{\rightarrow} \mathscr{I}_{C, X}^{2}(3) \otimes \operatorname{det} \mathscr{E}$, индуцирующий отображение $H^{1}\left(\mathscr{E} \otimes \mathscr{I}_{C, X}(2)\right) \rightarrow H^{1}\left(\mathscr{I}_{C, X}^{2}(3) \otimes \operatorname{det} \mathscr{E}\right)$, которое в композиции с $\gamma_{1}$ дает изоморфизм

$$
\gamma_{2}: \operatorname{Ext}_{Y}^{2}(\mathscr{E}, \mathscr{E}) \stackrel{\sim}{\rightarrow} H^{1}\left(\mathscr{I}_{C, X}^{2}(3) \otimes \operatorname{det} \mathscr{E}\right), \quad \gamma_{2} \sim\left(h, s^{2}\right) .
$$

Точные тройки $0 \rightarrow \mathscr{I}_{C, H} \stackrel{\cdot f_{Y}}{\longrightarrow} \mathscr{I}_{C, H}^{2}(3) \rightarrow \mathscr{I}_{C, X}^{2}(3) \rightarrow 0$ и $0 \rightarrow \mathscr{I}_{C, H} \rightarrow \mathscr{O}_{H} \rightarrow$ $\mathscr{O}_{C} \rightarrow 0$ вместе с $(2.9)$ и двойственностью Серра на $C$ дают цепочку канонических изоморфизмов:

$$
\begin{aligned}
H^{1}\left(\mathscr{I}_{C, X}^{2}(3) \otimes \operatorname{det} \mathscr{E}\right) & \simeq H^{2}\left(\mathscr{I}_{C, H} \otimes \operatorname{det} \mathscr{E}\right) \simeq H^{1}\left(\operatorname{det} \mathscr{E} \otimes \mathscr{O}_{C}\right) \\
& \simeq H^{0}\left((\operatorname{det} \mathscr{E})^{\vee} \otimes \omega_{C}\right)^{\vee}
\end{aligned}
$$

Далее, отображение $\phi_{h}: \mathscr{O}_{X}(1) \stackrel{\sim}{\rightarrow} \mathscr{N}_{X / Y}, \phi_{h} \sim h$, в композиции с $\psi: \mathscr{N}_{C / X} \stackrel{\sim}{\rightarrow}$ $\left.\mathscr{E}(1)\right|_{C}, \psi \sim s$, и каноническими изоморфизмами $\omega_{C} \simeq \omega_{Y} \otimes \mathscr{N}_{X / Y} \otimes \operatorname{det} \mathscr{N}_{C / X}$, $\omega_{Y} \simeq \mathscr{O}_{Y}(-3)$ дает следующий изоморфизм: $\chi:\left.\omega_{C} \stackrel{\sim}{\rightarrow} \operatorname{det} \mathscr{E}\right|_{C}, \chi \sim\left(h, s^{2}\right)$. Взяв его композицию с (2.13), получаем изоморфизм

$$
\begin{aligned}
\theta: H^{1}\left(\mathscr{I}_{C, X}^{2}(3) \otimes \operatorname{det} \mathscr{E}\right) & \stackrel{\text { can }}{\simeq} H^{0}\left((\operatorname{det} \mathscr{E})^{\vee} \otimes \omega_{C}\right)^{\vee} \stackrel{\sim}{\rightarrow} \\
& \stackrel{\sim}{\rightarrow} H^{0}\left(\left.\left(\left.\operatorname{det} \mathscr{E}\right|_{C}\right)^{\vee} \otimes \operatorname{det} \mathscr{E}\right|_{C}\right) \stackrel{\text { can }}{\simeq} \mathbb{C}, \\
\theta & \sim\left(h^{-1}, s^{-2}\right) .
\end{aligned}
$$

Далее, композиция изоморфизмов (2.12) и (2.14) дает требуемый изоморфизм $\alpha$ в (2.7) с $\alpha \sim\left(h^{0}, s^{0}\right)$, т. е. $\alpha$ не зависит от выбора скалярных множителей в $h$ и $s$. Таким образом, чтобы показать, что $\alpha$ - канонический изоморфизм, достаточно проверить, что $\alpha$ не зависит от выбора точки $z=\mathbb{C} s \in \mathbb{P}\left(H^{0}(\mathscr{E}(1))\right)$. Последнее утверждение очевидно, поскольку по построению изоморфизм $\alpha$ определен для любой точки ${ }^{2} z \in \mathbb{P}\left(H^{0}(\mathscr{E}(1))\right)$, тем самым задается морфизм тривиальных расслоений $\alpha: \operatorname{Ext}_{Y}^{2}(\mathscr{E}, \mathscr{E}) \otimes \mathscr{O}_{\mathbb{P}\left(H^{0}(\mathscr{E}(1))\right)} \rightarrow \mathbb{C} \otimes \mathscr{O}_{\mathbb{P}\left(H^{0}(\mathscr{E}(1))\right)}$. Имеем $\alpha \in H^{0}\left(\mathscr{O}_{\mathbb{P}\left(H^{0}(\mathscr{E}(1))\right)}\right)$

\footnotetext{
${ }^{1}$ Обозначаем это следующим образом: $\gamma_{1} \sim(h, s)$.

${ }^{2} \mathrm{~B}$ самом деле, для любого $z=\mathbb{C} s \in \mathbb{P}\left(H^{0}(\mathscr{E}(1))\right)$ из стабильности и локальной свободы $\mathscr{E}$ следует, что схема $C=(s)_{0}$ нулей сечения $s$ есть локально полное пересечение кораз-

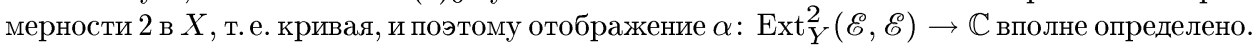


следовательно, морфизм $\alpha$ постоянен как функщия на проективном 5-мерном пространстве $\mathbb{P}\left(H^{0}(\mathscr{E}(1))\right)$.

Касательное распределение слоев проекции $p$ есть векторное расслоение $\mathscr{T}^{1,0}$, и его изотропность была доказана в предложении 2.1. Отсюда следует, что слои $p$ являются лагранжевыми.

\section{§ 3. Симплектическая структура на $\mathbf{J}_{Y}$}

В этом параграфе через $Y$ будем обозначать неособую 4 -мерную кубику в $\mathbb{P}^{5}$, через $h: \mathbf{J}=\mathbf{J}_{Y} \rightarrow U$ - относительный промежуточный якобиан семейства $\mathbf{X} \rightarrow$ $U \subset \check{\mathbb{P}}^{5}$ неособых гиперплоских сечений над открытым подмножеством $U$ в двойственном проективном пространстве $\check{\mathbb{P}}^{5}$. Через $X$ будем обозначать произвольное гладкое гиперплоское сечение $H \cap Y$ для $H \in U$, через $\mathbf{X}$ - схему нулей сечения пучка $\mathscr{O}_{\Pi}(3,0)$ на дивизоре инциденции $\Pi=\left\{(x, H) \in \mathbb{P}_{U}^{5} \mid x \in H\right\}$, где $\mathbb{P}_{U}^{5}=\mathbb{P}^{5} \times U \subset \mathbb{P}^{5} \times \check{\mathbb{P}}^{5}$. Можно зафиксировать это сечение, равно как и кубическую форму, определяющую $Y$ в $\mathbb{P}^{5}$, и рассматривать все изоморфизмы как зависящие только от этих данных.

Согласно работе $[5,8.22] \mathbf{J}$ имеет естественную (заданную с точностью до скалярного множителя) симплектическую структуру $\alpha_{\mathbf{J}} \in H^{0}\left(\mathbf{J}, \Omega_{\mathbf{J}}^{2}\right)$, для которой $h-$ лагранжево расслоение, т. е. слои $J_{X}$ проекции $h$ - промежуточные якобианы гиперплоских сечений $X=H \cap Y$-являются лагранжевыми подмногообразиями Ј. Идея состоит в том, чтобы восстановить симплектическую структуру из изоморфизмов $\mathscr{N}_{L / \mathbf{J}} \simeq \Omega_{L}^{1}$, которые она определяет для лагранжевых подмногообразий $L$. Для любой симплектической структуры $\alpha$ на $\mathbf{J}$, для которой $h$ - лагранжево расслоение, вышеуказанные изоморфизмы для лагранжевых подмногообразий $L=J_{X}$ склеиваются в изоморфизм векторных расслоений $\sigma(\alpha): \mathscr{T}_{U} \rightarrow h_{*} \Omega_{\mathbf{J} / U}^{1}$.

Обратно, пусть $\sigma \in \operatorname{Hom}\left(\mathscr{T}_{U}, h_{*} \Omega_{\mathbf{J} / U}^{1}\right)=H^{0}\left(U, \Omega_{U}^{1} \otimes h_{*} \Omega_{\mathbf{J} / U}^{1}\right)-$ произвольньй изоморфизм. Естественная точная тройка

$$
0 \rightarrow h^{*} \Omega_{U}^{1} \rightarrow \Omega_{\mathbf{J}}^{1} \rightarrow \Omega_{\mathbf{J} / U}^{1} \rightarrow 0
$$

влечет другую тройку, получаемую действием $\wedge^{2}$ :

$$
0 \rightarrow h^{*} \Omega_{U}^{2} \rightarrow \mathscr{F} \rightarrow h^{*} \Omega_{U}^{1} \otimes \Omega_{\mathbf{J} / U}^{1} \rightarrow 0,
$$

где $\mathscr{F} \subset \Omega_{\mathbf{J}}^{2}$ - векторное подрасслоение сечений, для которых $\mathscr{T}_{\mathbf{J}} / U-$ изотропное распределение. Пусть $\delta: H^{0}\left(\mathbf{J}, h^{*} \Omega_{U}^{1} \otimes \Omega_{\mathbf{J} / U}^{1}\right) \rightarrow H^{1}\left(\mathbf{J}, h^{*} \Omega_{U}^{2}\right)-$ связываюший гомоморфизм, определенный точной тройкой (3.2). Следующее утверждение очеВИДНО.

Лемма 3.1. Элемент $h^{*} \sigma$ поднимается в сечение $\alpha \in H^{0}(\mathbf{J}, \mathscr{F})$, если и только если $\delta\left(h^{*} \sigma\right)=0$. Подгем $\alpha$ определен с точностью до прибавления 2 -формы $h^{*} \beta$, где $\beta \in H^{0}\left(U, \Omega_{U}^{2}\right)$. Подбем становится однозначным, если наложить условие, что нулевое сечение $O \subset B$-лагранжево подмногообразие (m. e. $\left.\left.\alpha\right|_{O} \equiv 0\right)$.

Сформулируем результат Донаги-Маркмана в форме, удобной для наших целей. Пусть $Z$ - “схема Фано” кубики $Y$, т. е. схема Гильберта, параметризующая 
все прямые на $Y$. Она является гладким проективным 4-мерным многообразием. Бовиль и Донаги [2] доказали, что $Z$ является неприводимым симплектическим многообразием, деформационно эквивалентным 4-мерному многообразию Фуджики-Бовиля $S^{[2]}$ для К3-поверхности $S$. Пусть $\alpha_{Z}$ - его симплектическая структура, единственная с точностью до пропорциональности. Пусть $\pi: \mathbf{F} \rightarrow U-$ относительная поверхность Фано для $\mathbf{X}$ над $U$, т. е. семейство поверхностей $F(X)$, параметризуюших прямые в гиперплоских сечениях $X=H \cap Y$, где $H \in U$. По результатам [4] $\mathbf{J} / U$ канонически изоморфно относительному многообразию Альбанезе $\operatorname{Alb}(\mathbf{F} / U)$ и многообразию $\operatorname{Pic}^{0}(\mathbf{F} / U)$. Согласно работе [16] поверхности $\Phi$ ано $F(X)$ являются лагранжевыми в $Z$ и образуют открытое подмножество схемы Гильберта многообразия $Z$. В силу теоремы из [5] относительное многообразие Пикара $P=\operatorname{Pic}(\mathbf{L} / B)$ универсального семейства лагранжевых подмногообразий гладкого проективного симплектического многообразия $V$ над открытым множеством $B \subset \operatorname{Hilb}(V)$ имеет также симплектическую структуру, для которой $P / B-$ лагранжево расслоение. Применение этого результата к семейству $\mathbf{F} / U$ дает симплектическое 10-мерное многообразие $\operatorname{Pic}^{0}(\mathbf{F} / U)=\operatorname{Alb}(\mathbf{F} / U)=\mathbf{J}$.

Теорема (Донаги-Маркмана). Для любого $H \in U$ nусть $X=Y \cap H$, $F=F(X), \quad \sigma_{H}: \mathscr{N}_{F / Z} \rightarrow \Omega_{F}^{1}-$ изоморфизм, индуцированный формой $\alpha_{Z}$, и $H^{0}\left(\sigma_{H}\right): H^{0}\left(\mathscr{N}_{F / Z}\right) \rightarrow H^{0}\left(\Omega_{F}^{1}\right)$ - индуцированное отображение на глобальных сечениях. Ввиду естественных отождествлений

$$
H^{0}\left(F, \mathscr{N}_{F / Z}\right)=T_{[F]} \operatorname{Hilb}(Z)=T_{[F]} U, \quad H^{0}\left(F, \Omega_{F}^{1}\right)=\left.\Omega_{\mathrm{Alb}(F)}^{1}\right|_{0}=\left.\Omega_{J(X)}^{1}\right|_{0}
$$

пусть $\sigma: \mathscr{T}_{U} \rightarrow h_{*} \Omega_{\mathbf{J} / U}^{1}-$ изоморфизм, полученный релятивизацией конструкиии пространства $H^{0}\left(\sigma_{H}\right)$ над $U$. Тогда $\sigma$ удовлетворяет предположению леммы 3.1 и, следовательно, поднимается в единственную симплектическую структуру $\alpha_{\mathbf{J}}$ на $\mathbf{J}$, для которой слои и нулевое сечение проекции $h$ являются лагранжевыми подмногообразиями.

Теперь наша цель - идентифицировать $\sigma$ как канонический изоморфизм, построенньй в лемме 3.2 (см. ниже).

Пусть $\mathrm{pr}_{1}, \mathrm{pr}_{2}$ - естественные проекции на сомножители в $\mathbb{P}^{5} \times U$ и $\mathscr{O}(i, j)=$ $\operatorname{pr}_{1}^{*} \mathscr{O}_{\mathbb{P}^{5}}(i) \otimes \operatorname{pr}_{2}^{*} \mathscr{O}_{\breve{T} 5}(j)$

ЛЕмма 3.2. Имеют место канонические изоморфизмы

$$
h_{*} \Omega_{\mathbf{J} / U}^{1}=\pi_{*} \Omega_{\mathbf{F} / U}^{1}=R^{1} \operatorname{pr}_{2 *} \Omega_{\mathbf{X} / U}^{2}=\operatorname{pr}_{2 *} \mathscr{O}_{\mathbf{X}}(1,1)=\mathscr{T}_{U} .
$$

ДокАЗАТЕЛЬСТво. Первые два изоморфизма следуют из определений промежуточного якобиана и многообразия Альбанезе, равно как и отождествление $\mathbf{J}=$ $\operatorname{Alb}(\mathbf{F} / U)$, упомянутое выше. Третий изоморфизм является относительной версией формулы для касательного расслоения промежуточного якобиана 3 -мерной кубики. Чтобы это доказать, запишем точную последовательность относительных конормальных расслоений пары $\mathbf{X} \subset$ П над $U$, возведенную в третью внешнюю степень и подкрученную на $\mathscr{N}_{\mathbf{X} / \Pi}$ :

$$
0 \rightarrow \Omega_{\mathbf{X} / U}^{2} \rightarrow \Omega_{\Pi / U}^{3} \otimes \mathscr{N}_{\mathbf{X} / \Pi} \rightarrow \Omega_{\mathbf{X} / U}^{3} \otimes \mathscr{N}_{\mathbf{X} / \Pi} \rightarrow 0
$$


Применяя $R^{i} \mathrm{pr}_{2 *}$ и используя формулы Ботта, получаем канонический изоморфизм

$$
\operatorname{pr}_{2 *}\left(\Omega_{\mathbf{X} / U}^{3} \otimes \mathscr{N}_{\mathbf{X} / \Pi}\right) \stackrel{\sim}{\rightarrow} R^{1} \operatorname{pr}_{2 *} \Omega_{\mathbf{X} / U}^{2}
$$

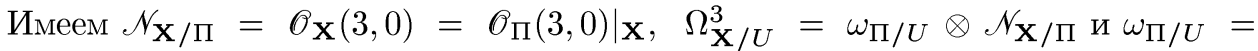
$\mathscr{O}_{\Pi}(-5,1)$, поэтому оба пучка в $(3.3)$ канонически изоморфны $\operatorname{pr}_{2 *} \mathscr{O}_{\mathbf{X}}(1,1)$.

Хорошо известно также, что дивизор инциденции П отождествляется с проективизированным кокасательным расслоением к $U: \Pi=\mathbb{P}\left(\mathscr{T}_{U}^{\vee}\right)$, следовательно, имеют место канонические изоморфизмы $\mathscr{T}_{U}^{\vee}=\operatorname{pr}_{2 *} \mathscr{O}_{\Pi / U}(1)=\operatorname{pr}_{2 *} \mathscr{O}_{\Pi}(1,1)=$ $\mathrm{pr}_{2 *} \mathscr{O}_{\mathbf{X}}(1,1)$.

Теорема Клеменса-Гриффитса о касательном расслоении для поверхности Фано $F(X)$ 3-мерной кубики $X$ утверждает, что имеет место изоморфизм $\mathscr{T}_{F(X)} \simeq$ $\left.\mathscr{S}_{G\left(1, \mathbb{P}^{4}\right)}\right|_{F(X)}$, где $G\left(1, \mathbb{P}^{n}\right)$ - грассманиан прямых в $\mathbb{P}^{n}$, а $\mathscr{S}_{G\left(1, \mathbb{P}^{n}\right)}-$ универсальное векторное расслоение ранга 2 на нем. Мы собираемся релятивизировать этот результат, чтобы вычислить пучок $\Omega_{\mathbf{F} / U}^{1}$.

Пусть $G=G\left(1, \mathbb{P}^{5}\right), \mathscr{S}_{G}$ - универсальное векторное расслоение, $\mathbb{P}^{5} \stackrel{q_{G}}{\leftarrow} \Gamma_{G} \stackrel{p_{G}}{\rightarrow}$ $G$ - универсальное семейство прямых, $Y \stackrel{q}{\leftarrow} \Gamma \stackrel{p}{\rightarrow} Z$ - его ограничение на прямые в $Y$ и $\mathbf{X} \stackrel{\mathbf{q}}{\leftarrow} \mathbf{\Gamma} \stackrel{\mathbf{p}}{\rightarrow} \mathbf{F}$ - релятивизация над $U$. Пусть $p_{i}-$ проекция на три сомножителя в $\mathbb{P}^{5} \times \check{\mathbb{P}}^{5} \times G$ и $\mathscr{O}(i, j, k)=p_{1}^{*} \mathscr{O}(i) \otimes p_{2}^{*} \mathscr{O}(j) \otimes p_{3}^{*} \mathscr{O}(k)$. Обозначим через $\Gamma_{u}, F_{u}$, $X_{u}, H_{u}=\mathbb{P}_{u}^{4}$ слои над $u \in U$ семейств $\boldsymbol{\Gamma}, \mathbf{F}, \mathbf{X}$, П соответственно.

ЛЕмма 3.3. Имеется канонический изоморфизм $\mathscr{T}_{\mathbf{F} / U}=\left.\left(\mathscr{O}_{\breve{\mathbb{P} 5}}(-1) \bigotimes \mathscr{S}_{G}\right)\right|_{\mathbf{F}}$, где операция $\square$ дает пучок на многообразии $\check{\mathbb{P}}^{5} \times G$, содержащем естественныцм образом семейство $\mathbf{F}$.

ДоказАТЕЛЬСТво. Естественные вложения $\boldsymbol{\Gamma} \subset \mathbf{X} \times_{U} \mathbf{F} \subset \Pi \times_{U} \mathbf{F}$ дают следующие точные последовательности:

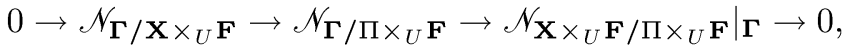

$$
\begin{aligned}
& 0 \rightarrow \mathscr{T}_{\boldsymbol{\Gamma} / \mathbf{F}} \rightarrow\left(p_{1} \times p_{2}\right)^{*} \mathscr{T}_{\Pi / U} \mid \boldsymbol{\Gamma} \rightarrow \mathscr{N}_{\boldsymbol{\Gamma} / \Pi \times_{U} \mathbf{F}} \rightarrow 0 .
\end{aligned}
$$

Имеем $\mathscr{T}_{\boldsymbol{\Gamma} / \mathbf{F}}=\left(p_{1} \times p_{3}\right) * \mathscr{T}_{\Gamma_{G} / G}$. Последовательность Эйлера

$$
0 \rightarrow \mathscr{O} \rightarrow q_{G}^{*} \mathscr{O}_{\mathbb{P}^{5}}(1) \otimes p_{G}^{*} \mathscr{S}_{G} \rightarrow \mathscr{T}_{\Gamma_{G} / G} \rightarrow 0
$$

влечет $\operatorname{det} \mathscr{T}_{\Gamma_{G} / G}=q_{G}^{*} \mathscr{O}_{\mathbb{P} 5}(1) \otimes p_{G}^{*} \mathscr{O}_{G}(-1)$. Находим последовательно детерминанты:

$$
\begin{aligned}
& \operatorname{det} \mathscr{T}_{\boldsymbol{\Gamma} / \mathbf{F}}=\mathscr{O}_{\boldsymbol{\Gamma}}(2,0,-1),\left.\quad \operatorname{det}\left(p_{1} \times p_{2}\right)^{*} \mathscr{T}_{\Pi / U}\right|_{\boldsymbol{\Gamma}}=\mathscr{O}_{\boldsymbol{\Gamma}}(5,-1,0), \\
& \operatorname{det} \mathscr{N}_{\boldsymbol{\Gamma} / \Pi \times_{U} \mathbf{F}}=\mathscr{O}_{\boldsymbol{\Gamma}}(3,-1,1) \text {, }
\end{aligned}
$$

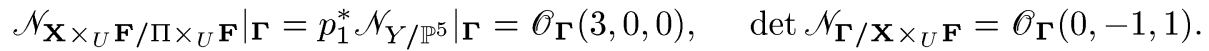

Дуализируя (3.4) и умножая тензорно на $\operatorname{det} \mathscr{N}_{\boldsymbol{\Gamma}} \mathbf{X} \times_{U} \mathbf{F}$, получаем точную тройку

$$
0 \rightarrow \mathscr{O}_{\boldsymbol{\Gamma}}(-3,-1,1) \rightarrow \mathscr{N}_{\mathbf{\Gamma} / \Pi \times_{U} \mathbf{F}}^{\vee}(0,-1,-1) \rightarrow \mathscr{N}_{\boldsymbol{\Gamma} / \mathbf{X} \times{ }_{U} \mathbf{F}} \rightarrow 0
$$


которая дает канонический изоморфизм

$$
\mathbf{p}_{*} \mathcal{N}_{\boldsymbol{\Gamma} / \mathbf{X} \times_{U} \mathbf{F}} \stackrel{\sim}{\rightarrow} R^{1} \mathbf{p}_{*} \mathscr{O}_{\boldsymbol{\Gamma}}(-3,-1,1)
$$

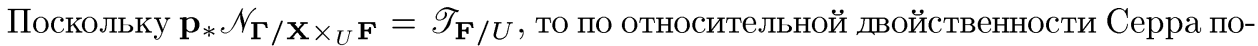
лучаем канонический изоморфизм

$$
\mathscr{T}_{\mathbf{F} / U}=\left(\mathbf{p}_{*} \mathscr{O}_{\boldsymbol{\Gamma}}(1,1,0)\right)^{\vee}
$$

Естественное отождествление $\mathscr{S}_{G}=\left(p_{G *} q_{G}^{*} \mathscr{O}_{\mathbb{P} 5}(1)\right)^{\vee}$ индуцирует канонический изоморфизм $\left(\mathbf{p}_{*} \mathscr{O}_{\boldsymbol{\Gamma}}(1,1,0)\right)^{*}=\left.\left(\mathscr{O}_{\check{\mathbb{P}}^{5}}(-1) \otimes \mathscr{S}_{G}\right)\right|_{\mathbf{F}}$, из которого следует утверждение леммы.

Следующая лемма релятивизирует изоморфизм $\left.\mathscr{N}_{F_{u} / Z} \simeq \mathscr{S}_{G}^{\vee}\right|_{F_{u}}$, вытекающий из равенства $\mathscr{N}_{G\left(1, \mathbb{P}_{u}^{4}\right) / G\left(1, \mathbb{P}^{5}\right)}=\left.\mathscr{S}_{G}^{\vee}\right|_{G\left(1, \mathbb{P}_{u}^{4}\right)}$.

ЛЕмма 3.4. Имеют место канонические изоморфизмы

$$
\mathscr{N}_{\mathbf{F} / \check{\mathbb{P}} 5 \times Z}=\left.\mathscr{O}_{\check{\mathbb{P}} 5}(1) \otimes \mathscr{S}_{G}^{\vee}\right|_{\mathbf{F}}=\Omega_{\mathbf{F} / U}^{1}
$$

Более того, применяя $\pi_{*}$, получаем изоморфизм $\pi_{*} \mathscr{N}_{\mathbf{F} / \check{\mathbb{P}}^{5} \times Z}=\pi_{*} \Omega_{\mathbf{F} / U}^{1}$, совпадающий с изоморфизмом леммы 3.2 ввиду естественного отождествления $\pi_{*} \mathscr{N}_{\mathbf{F} / \check{\mathbb{P}}^{5} \times Z}=\mathscr{T}_{U}$, вытекающего из интерпретации $\mathbf{F} / U$ как универсального семейства над открытым множеством $U \subset \operatorname{Hilb}(Z)$.

ДокАЗАТЕЛЬСТво. Представим $\mathbf{F}$ как полное пересечение $\boldsymbol{\sigma}_{\mathbf{1 1}} \cap(U \times Z)$, где

$$
\sigma_{11}=\left\{(u, l) \in \check{\mathbb{P}}^{5} \times G \mid l \subset H_{u}\right\}
$$

- универсальное шубертово многообразие $\sigma_{11}$ над $\check{\mathbb{P}}^{5}$. Это - схема нулей сечения $\left(q_{G} \times \mathrm{id}\right)_{*}\left(p_{G} \times \mathrm{id}\right)^{*} \tau_{0}$, где $\tau_{0} \in H^{0}\left(\mathbb{P}^{5} \times \check{\mathbb{P}}^{5}, \mathscr{O}_{\mathbb{P}^{5} \times \check{\mathbb{P}}^{5}}(1,1)\right)$, а $\tau_{0}=0$ - уравнение дивизора П. Так как $\left(q_{G} \times \mathrm{id}\right)_{*}\left(p_{G} \times \mathrm{id}\right)^{*} \mathscr{O}_{\mathbb{P}^{5} \times \check{\mathbb{P}}^{5}}(1,1)=\mathscr{O}_{\breve{\mathbb{P}}^{5}}(1) \otimes \mathscr{S}_{G}^{\vee}$, то получаем доказательство первого утверждения.

Второе утверждение следует немедленно из вычисления Клеменса-Велтерса дифференциала отображения Абеля-Якоби (см. $[17, \S 2]$ либо диаграмму (5.5) в $§ 5)$.

\section{§4. Симплектическая структура} на многообразии Фано $Z$ четырехмерной кубики $Y$

Рассмотрим конструкцию симплектической структуры. Пусть $\overline{\mathbf{F}}$ и $\overline{\boldsymbol{\Gamma}}$ - замыкание $\mathbf{F}$ в $\check{\mathbb{P}}^{5} \times G$ и $\boldsymbol{\Gamma}$ в $\mathbb{P}^{5} \times \check{\mathbb{P}}^{5} \times G$ соответственно.

Имеем диаграмму естественных вложений

$$
\begin{array}{r}
Y \times \overline{\mathbf{F}} \subset \mathbb{P}^{5} \times \overline{\mathbf{F}} \\
\cup \quad \cup \\
\overline{\mathbf{\Gamma}} \subset \mathbf{X} \times_{\check{\mathbb{P}} 5} \overline{\mathbf{F}} \subset \Pi \times_{\check{\mathbb{P}} 5} \overline{\mathbf{F}}
\end{array}
$$


Заметим, что $\overline{\mathbf{F}}$ и $\overline{\boldsymbol{\Gamma}}$-неособые многообразия размерностей 7 и 8 соответственно. Это следует из того факта, что $\overline{\mathbf{F}}$ допускает естественный гладкий морфизм на $Z$ со слоями $\mathbb{P}^{3}$, а $\overline{\boldsymbol{\Gamma}} / \overline{\mathbf{F}}$ гладко со слоем $\mathbb{P}^{1}$. Выпишем точные последовательности нормальных расслоений:

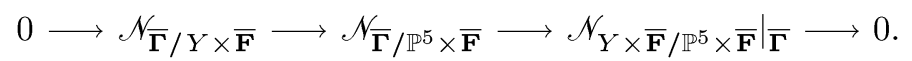

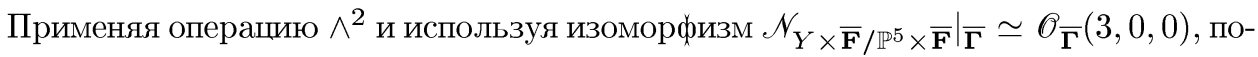
лучаем точную тройку

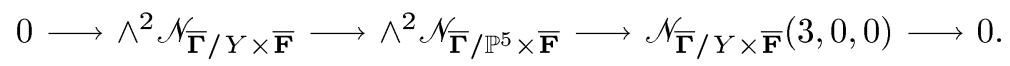

Теперь из доказательства леммы 3.3 следует, что

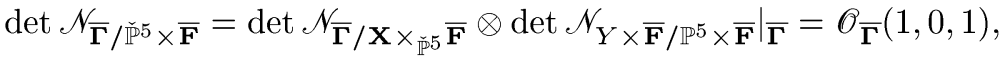

$$
\begin{aligned}
& \omega_{\bar{\Gamma} / \overline{\mathbf{F}}}=\mathscr{O}_{\bar{\Gamma}}(-2,0,1) .
\end{aligned}
$$

Используя эти формулы и подкручивая последнюю точную тройку на $\mathscr{O} \overline{\boldsymbol{\Gamma}}(-3,0,0)$, получаем

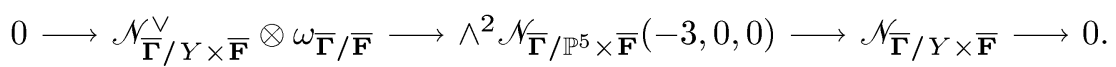

ЗАмечание 4.1. Рассмотрим коммутативньй квадрат

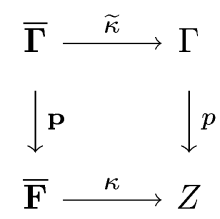

где $\widetilde{\kappa}$ - ограничение на $\overline{\boldsymbol{\Gamma}}$ естественной проекции $\mathbb{P}^{5} \times \check{\mathbb{P}}^{5} \times G \rightarrow \mathbb{P}^{5} \times G$. Тогда (4.4) получается применением $\widetilde{\kappa}^{*}$ к тройке

$$
0 \rightarrow \mathscr{N}_{\Gamma / Y \times Z}^{\vee} \otimes \omega_{\Gamma / Z} \rightarrow \wedge^{2} \mathscr{N}_{\Gamma / \mathbb{P}^{5} \times Z}(-3,0) \rightarrow \mathscr{N}_{\Gamma / Y \times Z} \rightarrow 0 .
$$

Для любой точки $z \in \overline{\mathbf{F}}$, соответственно, $l \in Z$, имеем

$$
\wedge^{2} \mathscr{N}_{\overline{\mathbf{\Gamma}} / \mathbb{P}^{5} \times \overline{\mathbf{F}}}(-3,0,0) \mid \mathbf{p}^{-1}(z) \simeq \mathscr{O}_{\mathbb{P}^{1}}(-1)^{\oplus 6},
$$

соответственно,

$$
\wedge^{2} \mathscr{N}_{\Gamma / \mathbb{P}^{5} \times Z}(-3,0) \mid p^{-1}(l) \simeq \mathscr{O}_{\mathbb{P}^{1}}(-1)^{\oplus 6},
$$

поэтому замена базы влечет

$$
R^{i} \mathbf{p}_{*}\left(\wedge^{2} \mathscr{N}_{\overline{\boldsymbol{\Gamma}} / \mathbb{P}^{5} \times \overline{\mathbf{F}}}(-3,0,0)\right)=0, \quad R^{i} p_{*}\left(\wedge^{2} \mathscr{N}_{\Gamma / \mathbb{P}^{5} \times Z}(-3,0)\right)=0, \quad i \geqslant 0
$$


Применяя $R^{i} p_{*}$ к (4.6) и, соответственно, $R^{i} \mathbf{p}_{*}$ к (4.4) и используя (4.7), относительную двойственность Серра, замечание 4.2 и изоморфизм $p_{*} \mathscr{N}_{\Gamma / \mathbb{P}^{5} \times Z} \simeq \mathscr{T} Z$, получаем изоморфизмы

$$
\wedge_{Z}: \mathscr{T} Z \stackrel{\sim}{\rightarrow} \Omega_{Z}
$$

и, соответственно,

$$
\kappa^{*} \wedge_{Z}: \kappa^{*} \mathscr{T} Z \stackrel{\sim}{\rightarrow} \kappa^{*} \Omega_{Z} .
$$

Поскольку согласно [2] $Z$ есть неприводимое симплектическое многообразие, из принципа Бохнера для неприводимых симплектических многообразий [3] вытекает, что изоморфизм (4.8) есть симплектическая форма на $Z$. Таким образом, получаем явную конструкцию симплектической структуры на $Z$, формулируемую в следующей теореме.

ТЕОРема 4.2. Симплектическая структура на $Z$ есть связывающий гомоморфизм (4.8) в длинной точной последовательности функторов $R^{i} p_{*}$, ассоциированной с точной тройкой (4.6).

Напомним, что согласно результатам, полученным в предыдущем параграфе,

$$
\mathbf{p}_{*} \mathscr{N}_{\mathbf{\Gamma} / \mathbf{X} \times_{U} \mathbf{F}}=\mathscr{T} \mathbf{F} / U, \quad \mathbf{p}_{*} \mathscr{O}_{\boldsymbol{\Gamma}}(1,1,0)=\mathscr{N}_{\mathbf{F} / U \times Z}
$$

По относительной двойственности Серра имеем

$$
R^{1} \mathbf{p}_{*}\left(\mathscr{N}_{\mathbf{\Gamma} / \mathbf{X} \times_{U} \mathbf{F}}^{\vee} \otimes \omega_{\Gamma / \mathbf{F}}\right)=\Omega_{\mathbf{F} / U}, \quad R^{1} \mathbf{p}_{*}\left(\mathscr{O}_{\boldsymbol{\Gamma}}(1,1,0)^{\vee} \otimes \omega_{\boldsymbol{\Gamma} / \mathbf{F}}\right)=\mathscr{N}_{\mathbf{F} / U \times Z}^{\vee}
$$

Таким образом, используя (3.14), (3.15), (3.13) и применяя $R^{i} \mathbf{p}_{*}$ к $(4.4)$, получаем коммутативную диаграмму

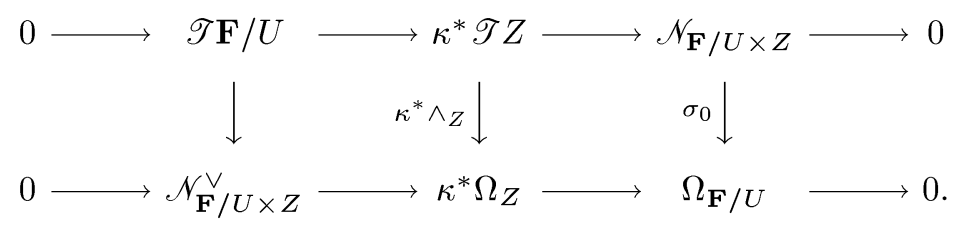

По теореме $4.3 \wedge_{Z}$ есть симплектическая форма; кроме того, по конструкции, $\sigma_{0}$ есть изоморфизм из леммы 3.4. Поэтому справедлива

Теорема 4.3. Коммутативная диаграмма (4.12) антиавтодуальна, и изоморфизм $\sigma_{0}$ в этой диаграмме совпадает с каноническим изоморфизмом леммы 3.4.

В частности, композиция $\mathscr{T} \mathbf{F} / U \rightarrow \kappa^{*} \mathscr{T} Z{\stackrel{\kappa}{*} \wedge^{Z}}^{*} \Omega_{Z} \rightarrow \Omega_{\mathbf{F} / U}$ в этой диаграмме есть нулевое отображение. Это дает другое доказательство результата Вуазен [16] о том, что поверхности Фано гиперплоских сечений $Y$ являются лагранжевыми подмногообразиями симплектической структуры на $Z$. 


\section{$\S 5$. Связь между симплектическими структурами на $\mathfrak{M}_{Y}$ и $\mathbf{J}_{Y}$}

5.1. Кодифференциал отображения Абеля-Якоби. Напомним следуюшую интерпретацию Клеменса-Велтерса кодифференциала $d \Phi^{\vee}$ отображения Абеля-Якоби $\Phi: B \rightarrow J(X)$, где $B$ - база некоторого семейства кривых на гладком гиперплоском сечении $X=Y \cap \mathbb{P}^{4}$ кубики $Y$ (см. $\left.[17, \S 2]\right)$. Пусть $[C]$ - точка в $B$, соответствуюшая кривой - локально полному пересечению $C \subset X$, и пусть

$y=\Phi(C)$. Применяя $\wedge^{3}$ к точной тройке $0 \rightarrow \mathscr{N}_{X / \mathbb{P}^{4}}^{\vee} \rightarrow \Omega_{\mathbb{P}^{4}}^{1} \mid X \rightarrow \Omega_{X}^{1} \rightarrow 0$ и подкручивая на $\mathscr{N}_{X / \mathbb{P}^{4}}=\mathscr{O}_{X}(3)$, получаем точную тройку

$$
0 \rightarrow \Omega_{X}^{2} \rightarrow \Omega_{\mathbb{P}^{4}}^{3} \otimes \mathscr{N}_{X / \mathbb{P}^{4}} \rightarrow \mathscr{O}_{X}(1) \rightarrow 0
$$

Переходя к когомологиям этой последовательности, получаем кограничный изоморфизм

$$
R: H^{0}\left(\mathscr{O}_{X}(1)\right) \stackrel{\sim}{\rightarrow} H^{1}\left(\Omega_{X}^{2}\right) .
$$

Заметим, что, по конструкции, $R$ есть ограничение на слой $\mathbb{P}^{4}$ изоморфизма $\mathrm{pr}_{2 *} \mathscr{O}_{\mathbf{X}}(1,1)=R^{1} \mathrm{pr}_{2 *} \Omega_{\mathbf{X} / U}^{2}$ из леммы 3.2 .

Далее, тройка (5.1), ограниченная на $C$, очевидно, включается в коммутативную диаграмму

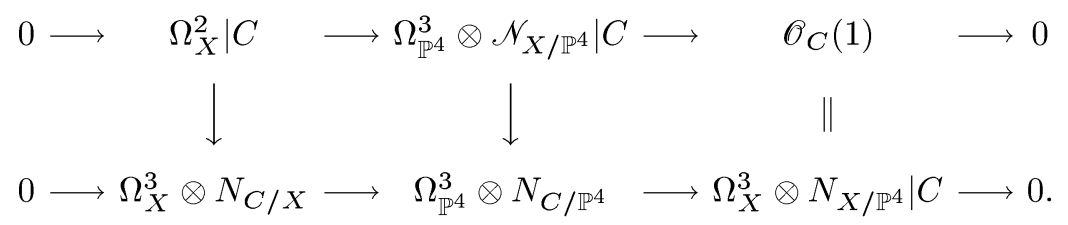

Очевидно, что для кривой (являющейся локально полным пересечением) $C \subset X$ верно:

$$
\Omega_{X}^{3} \otimes N_{C / X}=\omega_{X} \otimes N_{C / X}=\omega_{X} \otimes \operatorname{det} N_{C / X} \otimes N_{C / X}^{\vee}=\omega_{C} \otimes N_{C / X}^{\vee},
$$

так что по двойственности Серра $H^{1}\left(\Omega_{X}^{3} \otimes N_{C / X}\right)=H^{0}\left(N_{C / X}\right)^{\vee}$. Следовательно, переходя к когомологиям нижней тройки, получаем отображение

$$
\beta_{C}: H^{0}\left(\mathscr{O}_{C}(1)\right) \rightarrow H^{0}\left(N_{C / X}\right)^{\vee}
$$

Согласно $[17, \S 2]$ кодифференциал $\left.d \Phi^{\vee}\right|_{[C]}$ в точке $[C]$ отображения Абеля-Якоби $\Phi: B \rightarrow J(X)$ удовлетворяет коммутативной диаграмме

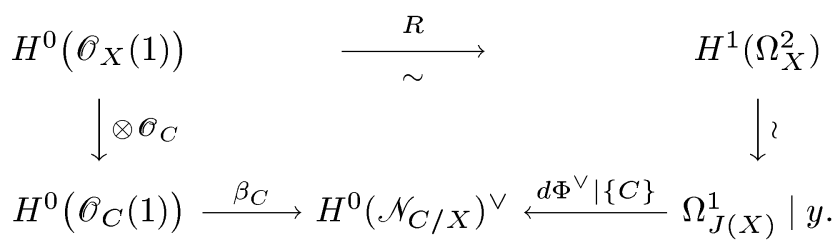


Теперь, как и в $\S 3$, пусть $U=\left\{\mathbb{P}^{4} \in \check{\mathbb{P}}^{5} \mid X=Y \cap \mathbb{P}^{4}\right.$ гладко $\}, h: \mathbf{J} \rightarrow U$ и $\pi: \mathbf{F} \rightarrow U$ - проекции, а $\sigma=\pi_{*} \sigma_{0}: \pi_{*} \mathscr{N}_{\mathbf{F} / \check{\mathbb{P}} 5 \times Z}\left|U \stackrel{\sim}{\rightarrow} \pi_{*} \Omega_{\mathbf{F} / \tilde{\mathbb{P}}^{5}}^{1}\right| U \simeq h_{*} \Omega_{\mathbf{J} / U}^{1}-$ канонический изоморфизм (см. леммы 3.2 и 3.4 ). Возьмем точку $y \in h^{-1}(U)$, и пусть $X=\mathbb{P}^{4} \cap Y$, где $\mathbb{P}^{4}=h(y)$. Заметим, что по лемме 3.4 имеется естественное отождествление $H^{0}\left(\mathscr{O}_{X}(1)\right) \simeq h^{*} \pi_{*} \mathscr{N}_{\mathbf{F} / \tilde{\mathbb{P}}^{5} \times Z} \mid y$. Эти изоморфизмы вместе с (5.5) и соответствующими изоморфизмами замены базы дают коммутативную диаграмму

$$
\begin{gathered}
H^{0}\left(\mathscr{O}_{X}(1)\right) \stackrel{\simeq}{\longrightarrow} h^{*} \pi_{*} \mathscr{N}_{\mathbf{F} / \check{\mathbb{P}}^{5} \times Z} \mid y \\
R \downarrow^{2} \quad 2 h^{*} \sigma \\
H^{1}\left(\Omega_{X}^{2}\right) \simeq \Omega_{\mathbf{J} / U}^{1}\left|y \simeq h^{*} h_{*} \Omega_{\mathbf{J} / U}^{1}\right| y .
\end{gathered}
$$

Здесь $H^{0}\left(\mathscr{O}_{X}(1)\right)=h^{*} \mathscr{T} U \mid y$, и изоморфизм $R$ глобализуется в изоморфизм

$$
\mathscr{R}: h^{*} \mathscr{T} U \stackrel{\sim}{\rightarrow} \Omega_{\mathbf{J} / U}^{1}
$$

Теперь рассмотрим симплектическую структуру Донаги-Маркмана $\wedge_{\mathbf{J}}: \mathscr{T} \mathbf{J} \stackrel{\sim}{\rightarrow}$ $\Omega_{\mathbf{J}}^{1}$, индуцируемую на $\mathbf{J}$ симплектической структурой $\wedge_{Z}$ на $Z$. Тогда, собирая вместе (5.6) и коммутативный квадрат в правой части (4.12), получаем следующую теорему.

ТЕОРема 5.1. Имеет место антиавтодуальная коммутативная диаграмма

$$
\begin{gathered}
0 \longrightarrow \mathscr{T} \mathbf{J} / U \longrightarrow{ }^{2} \mathbf{J} \longrightarrow h^{*} \mathscr{T} U \longrightarrow{ }^{2} \longrightarrow{ }^{2} \mid 2 \\
0 \longrightarrow h^{*} \Omega_{U}^{1} \longrightarrow \Omega_{\mathbf{J}}^{1} \longrightarrow
\end{gathered}
$$

5.2. Локальные подъемы структуры $\wedge$ J на $\mathfrak{M}$. Рассмотрим открытоеподмножество $\mathscr{H}^{\prime}=\left\{c \in \operatorname{Hilb}_{Y}^{5 n} \mid C\right.$ - гладкая проективно нормальная квинтика $\}$ в Hilb ${ }_{Y}^{5 n}$ с проекцией $\rho^{\prime}: \mathscr{H}^{\prime} \rightarrow \check{\mathbb{P}}^{5}: C \mapsto\langle C\rangle$. Пусть $Y$ - достаточно обшая четырехмерная кубика, так что согласно [11] $\mathscr{H}^{\prime}$ есть гладкое неприводимое открытое подмножество в $\operatorname{Hilb}_{Y}^{5 n}, \operatorname{dim} \mathscr{H}^{\prime}=15, \rho\left(\mathscr{H}^{\prime}\right)$ является плотным открытым подмножеством в $\check{\mathbb{P}}^{5}$, и сушествуют плотное открытое подмножество $U \subset \check{\mathbb{P}}^{5}$ и, соответственно, плотное открытое подмножество $\mathscr{H}$ в $\mathscr{H}^{\prime}, \mathscr{H} \subset \rho^{-1}(U)$, такие, что любое $X \in U$ есть гладкое гиперплоское сечение $Y$ и $\rho=\rho^{\prime} \mid \mathscr{H}: \mathscr{H} \rightarrow U$ есть гладкий морфизм относительной размерности 10. Для любой точки $C \in \mathscr{H}$ существует локальное аналитическое (или локальное в этальной топологии) сечение проекции $\rho$, проходящее через $C$, т. е. аналитическая (соответственно, этальная) окрестность $\mathscr{U} \ni \rho(C)$ и голоморфное (соответственно, регулярное) отображение $s=s_{\mathscr{U}}: \mathscr{U} \rightarrow \mathscr{H}$ такое, что $s(\mathscr{U}) \ni C$. Пусть $\mathscr{H}_{\mathscr{U}}=\rho^{-1}(\mathscr{U})$, $\mathbf{J}_{\mathscr{U}}=h^{-1}(\mathscr{U}), \mathfrak{M}_{\mathscr{U}}=p^{-1}(\mathscr{U})$, где $p: \mathfrak{M} \rightarrow \check{\mathbb{P}}^{5}: \mathscr{E} \rightarrow\langle\operatorname{Supp}(\mathscr{E})\rangle$ есть проекция, и обозначим $p_{\mathscr{U}}=\left.p\right|_{\mathfrak{M}_{\mathscr{U}}}$. Сечение $s$ определяет относительное отображение Абеля-Якоби $\Phi_{s}: \mathscr{H}_{\mathscr{U}} \rightarrow \mathbf{J}_{\mathscr{U}}: C \mapsto \Phi_{X, s}(C)$, где $\Phi_{X, s}: \rho^{-1}(X) \rightarrow J_{X}: C \mapsto \Phi(C-$ 
$s(X))$ есть обычное отображение Абеля-Якоби, а $J_{X}=h^{-1}(X)$. По [11, теорема 5.6] отображение $\Phi_{\mathscr{U}}$ обладает факторизацией Штейна $\Phi_{\mathscr{U}}=\Psi_{\mathscr{U}} \circ \phi \mathscr{U}$, где $\phi_{\mathscr{U}}: \mathscr{H}_{\mathscr{U}} \rightarrow \mathfrak{M}_{\mathscr{U}}$ - гладкое голоморфное отображение относительной размерности 5, а $\Psi_{\mathscr{U}}: \mathfrak{M}_{\mathscr{U}} \rightarrow \mathbf{J}_{\mathscr{U}}$ - этальное голоморфное отображение.

Далее, переходя к $\wedge^{2}$ в нижней тройке (5.8), получаем диаграмму

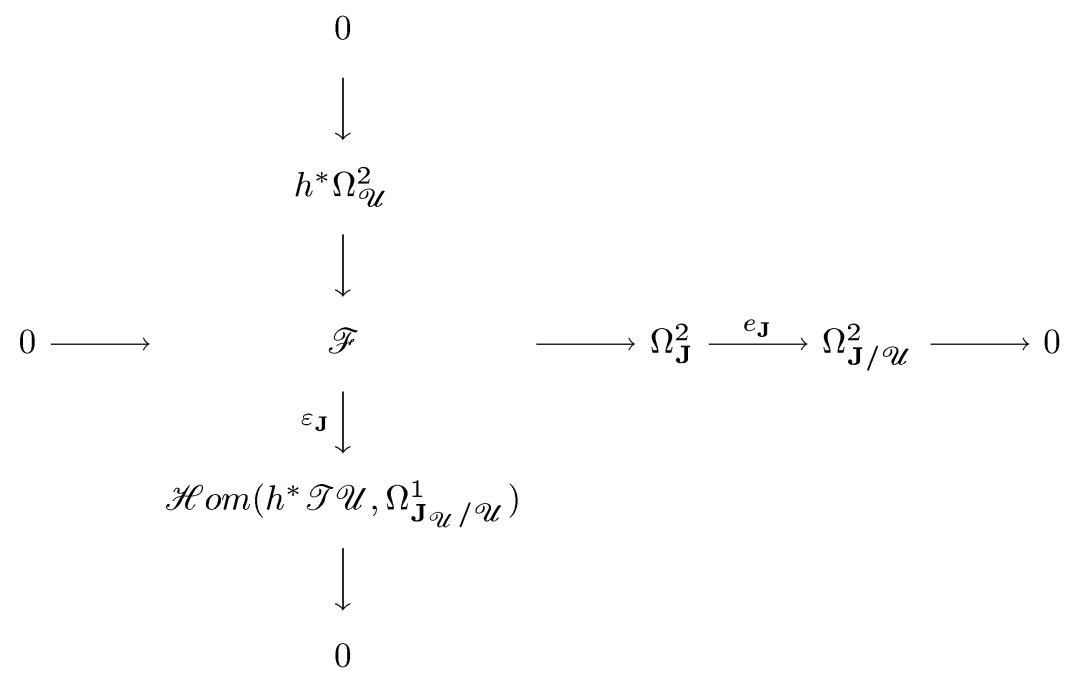

Аналогично, имеем на $\mathfrak{M}_{\mathscr{U}}$

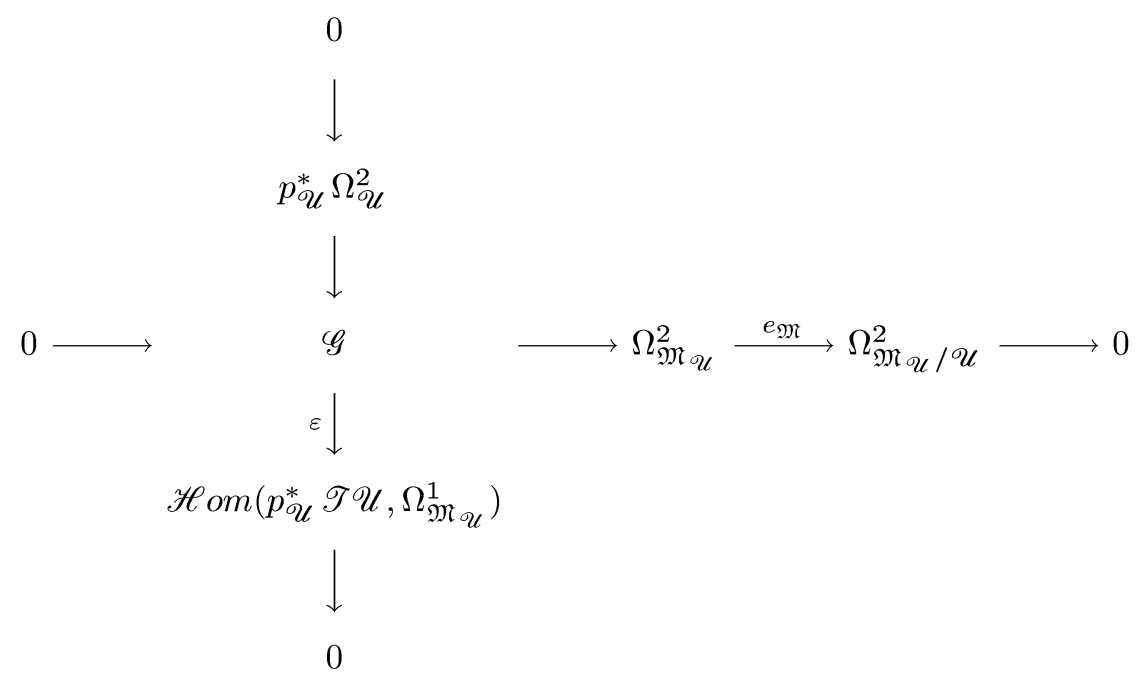

и поскольку $\Psi_{\mathscr{U}}: \mathfrak{M}_{\mathscr{U}} \rightarrow \mathbf{J}_{\mathscr{U}}$ этально, диаграмма (5.10) получается из (5.9) применением $\Psi_{\mathscr{U}}^{*}$ :

$$
(5.10)=\Psi_{\mathscr{U}}^{*}(5.9) .
$$

Теперь пусть $\Lambda_{\mathbf{J}} \in H^{0}\left(\Omega_{\mathbf{J}}^{2}\right)$ - симплектическая структура Донаги-Маркманана $\mathbf{J}_{\text {и }} \Lambda_{\mathbf{J}_{\mathscr{U}}}=\Lambda_{\mathbf{J}} \mid \mathfrak{M}_{\mathscr{U}}-$ ее ограничение на $\mathbf{J}_{\mathscr{U}}$, и пусть $\Lambda_{\mathfrak{M}_{\mathscr{U}}}=\Lambda \mid \mathfrak{M}_{\mathscr{U}}$, где $\Lambda$ - квазисимплектическая форма Ионеды, введенная ранее в предложении 2.1. Согласно 
предложению 2.1 для любого $[\mathscr{E}] \in \mathfrak{M}_{\mathscr{U}}$ векторное пространство $T_{[\mathscr{E}]} \mathfrak{M}_{\mathscr{U}} / \mathscr{U} \subset$ $T_{[\mathscr{E}]} \mathfrak{M}_{\mathscr{U}}$ изотропно относительно $\Lambda_{\mathfrak{M}}$, следовательно,

$$
e_{\mathfrak{M}}\left(\Lambda_{\mathfrak{M}}\right)=0
$$

По той же причине

$$
e_{\mathbf{J}}\left(\Lambda_{\mathbf{J}_{\mathscr{U}}}\right)=0 .
$$

Из условий (5.12) и (5.13) вытекает, что $\Lambda_{\mathfrak{M}} \in H^{0}(\mathscr{G})$ и, соответственно, $\Lambda_{\mathbf{J}_{\mathscr{U}}} \in H^{0}(\mathscr{F})$. Теперь докажем, что

$$
\varepsilon\left(\Lambda_{\mathfrak{M}_{\mathscr{U}}}\right)=\Psi_{\mathscr{U}}^{*} \varepsilon_{\mathbf{J}}\left(\Lambda_{\mathbf{J}_{\mathscr{U}}}\right)
$$

или, что то же самое в силу (5.11), что

$$
\varepsilon\left(\Lambda_{\mathfrak{M}_{\mathscr{U}}}-\Psi_{\mathscr{U}}^{*} \Lambda_{\mathbf{J}_{\mathscr{U}}}\right)=0
$$

Чтобы доказать (5.14), возьмем произвольное $[\mathscr{E}] \in \mathfrak{M}_{\mathscr{U}}$, и пусть $y=\Psi \mathscr{U}([\mathscr{E}])$, $X=h(y)$. В силу отождествлений $T_{[\mathscr{E}]} \mathfrak{M}_{\mathscr{U}} / \mathscr{U}=H^{1}(\mathscr{E} \otimes \mathscr{E}), p_{\mathscr{U}}^{*} \mathscr{T} \mathscr{U}_{[\mathscr{E}]}=$ $H^{0}\left(\mathscr{O}_{X}(1)\right)$ имеем изоморфизм $\sharp \Lambda:=\left.\varepsilon\left(\Lambda_{\mathfrak{M}_{\mathscr{U}}}\right)\right|_{[\mathscr{E}]}: H^{0}\left(\mathscr{O}_{X}(1)\right) \stackrel{\sim}{\rightarrow} H^{1}(\mathscr{E} \otimes \mathscr{E}) \vee$. По теореме 5.2 вместе с отождествлениями $T_{y} \mathbf{J}_{\mathscr{U}} / \mathscr{U}=H^{1}\left(\Omega_{X}^{2}\right)^{\vee},\left.h^{*} \mathscr{T} \mathscr{U}\right|_{y}=$ $H^{0}\left(\mathscr{O}_{X}(1)\right)$ имеем изоморфизм $R=\left.\varepsilon_{\mathbf{J}}\left(\Lambda_{\mathbf{J}_{\mathscr{U}}}\right)\right|_{y}: H^{0}\left(\mathscr{O}_{X}(1)\right) \stackrel{\sim}{\rightarrow} H^{1}\left(\Omega_{X}^{2}\right)$. Поэтому в силу (5.5) равенство (5.14) сводится к коммутативности диаграммы

$$
\begin{aligned}
& H^{1}\left(\Omega_{X}^{2}\right) \stackrel{d \Psi_{\mathscr{U}}^{\vee}}{\longrightarrow} H^{1}(\mathscr{E} \otimes \mathscr{E})^{\vee} \\
& R \uparrow_{2} \quad \nearrow_{\sharp \Lambda} \\
& H^{0}\left(\mathscr{O}_{X}(1)\right)
\end{aligned}
$$

т. е., после дуализации и использования (5.5), к коммутативности квадрата

$$
\begin{array}{rr}
H^{0}\left(\mathscr{N}_{C / X}\right) \stackrel{\left.d \Phi_{X, s}\right|_{[C]}}{\longrightarrow} H^{1}(\mathscr{E} \otimes \mathscr{E}) \\
\beta_{C}^{\vee} \downarrow & \left.\downarrow^{\sharp} \Lambda\right)^{\vee} \\
H^{0}\left(\mathscr{O}_{C}(1)\right)^{\vee} \stackrel{\sim}{\longrightarrow} H^{0}\left(\mathscr{O}_{X}(1)\right)^{\vee},
\end{array}
$$

где $C$ - любая кривая в $\phi_{\mathscr{U}}^{-1}([\mathscr{E}])$.

По лемме 2.2 и диаграмме (2.4) диаграмма (5.17) эквивалентна квадрату

$$
\begin{aligned}
& H^{0}\left(\mathscr{N}_{C / X}\right) \otimes H^{0}\left(\mathscr{O}_{C}(1)\right) \stackrel{m}{\longrightarrow} H^{0}\left(\mathscr{N}_{C / X}(1)\right) \\
&\left.d \Phi_{X, s}\right|_{[C]} \otimes\left(\mathrm{res}^{-1}\right) \downarrow \downarrow \widetilde{\delta} \\
& H^{1}(\mathscr{E} \otimes \mathscr{E}) \otimes H^{0}\left(\mathscr{O}_{X}(1)\right) \stackrel{m}{\longrightarrow} H^{1}(\mathscr{E} \otimes \mathscr{E}(1)),
\end{aligned}
$$


где $m$ - обычное умножение, $\widetilde{\delta}$ - композиция,

$$
\widetilde{\delta}: H^{0}\left(\mathscr{N}_{C / X}(1)\right) \stackrel{\delta}{\rightarrow} H^{1}\left(\mathscr{I}_{C, X}^{2}(3)\right) \underset{\sim}{\stackrel{\gamma_{1}}{\sim}} H^{1}\left(\mathscr{I}_{C, X}(2) \otimes \stackrel{\mathscr{E}}{\underset{\sim}{\gamma_{2}}} H^{1}(\mathscr{E} \otimes \mathscr{E}(1)),\right.
$$

$\delta$ - связывающее отображение в когомологиях точной тройки $0 \rightarrow \mathscr{I}_{C, X}^{2}(3) \rightarrow$ $\mathscr{I}_{C, X}(3) \rightarrow \mathscr{N}_{C / X}(1) \rightarrow 0$, а изоморфизмы $\gamma_{1}, \gamma_{2}$ получаются из троек $0 \rightarrow$ $\mathscr{O}_{X}(1) \rightarrow \mathscr{I}_{C, X}(2) \otimes \mathscr{E} \rightarrow \mathscr{I}_{C, X}^{2}(3) \rightarrow 0,0 \rightarrow \mathscr{E} \rightarrow \mathscr{E} \otimes \mathscr{E}(1) \rightarrow \mathscr{I}_{C, X}(2) \otimes \mathscr{E} \rightarrow 0$ с использованием равенств $h^{i}\left(\mathscr{O}_{X}(1)\right)=0, h^{i}(\mathscr{E})=0, i=1,2$. Коммутативность (5.18) очевидна. Следовательно, получаем (5.14).

Обозначим $\Lambda_{\mathscr{U}, s}=\Lambda_{\mathfrak{M}_{\mathscr{U}}}-\Psi_{\mathscr{U}}^{*} \Lambda_{\mathbf{J}_{\mathscr{U}}}$. Из (5.15) и (5.9) следует, что форма ${ }^{3}$ $\Lambda_{\mathscr{U}, s}$ принадлежит $H^{0}\left(p_{\mathscr{U}}^{*} \Omega_{\mathscr{U}}^{2}\right)$. Таким образом, получаем следуюшую теорему, связьвающую квазисимплектические структуры $\Lambda_{\mathfrak{M}_{\mathscr{U}}}$ и $\Lambda_{\mathbf{J}_{\mathscr{U}}}$.

Теорема 5.2. Для данных $(\mathscr{U}, s)$, обозначенных выше, существует 2-форма $\Lambda_{\mathscr{U}, s} \in H^{0}\left(p_{\mathscr{U}}^{*} \Omega_{\mathscr{U}}^{2}\right)$ такая, что

$$
\Lambda_{\mathfrak{M}_{\mathscr{U}}}=\Psi_{\mathscr{U}}^{*} \Lambda_{\mathbf{J}_{\mathscr{U}}}+\Lambda_{\mathscr{U}, s} .
$$

5.3. Симплектическая структура на $\mathfrak{M}$. Только в этом пункте мы воспользуемся следуюшим результатом из [8].

ТЕОРема 5.3. Для любой неособой трехмерной кубики $X$ отображение $\Psi: M_{X} \rightarrow J^{2}(X)$ есть открытое вложение.

ДокАЗАТЕЛЬСТво. См. теорему 3.2 и следствия $3.3,5.1$ из [8].

Из этой теоремы следует, что отображения $\Psi_{\mathscr{U}}: \mathfrak{M}_{\mathscr{U}} \rightarrow \mathbf{J}_{\mathscr{U}}$, определенные локальными сечениями $s \mathscr{U}: \mathscr{U} \rightarrow \mathscr{H}$ проекции $\rho: \mathscr{H} \rightarrow U$, также являются открытыми вложениями. Пусть $\left\{\mathscr{U}_{\alpha}\right\}_{\alpha \in I}$ - открытое покрытие $U$ в классической либо этальной топологии такое, что сушествуют локальные сечения $s_{\alpha}: \mathscr{U}_{\alpha} \rightarrow \mathscr{H}$ проекции $\rho$, и пусть $\sigma_{\alpha}: \mathscr{U}_{\alpha} \rightarrow \mathfrak{M}_{\mathscr{U}}-$ соответствующие сечения отображения $\Psi$. Тогда $c=\left(\sigma_{\alpha \beta}\right)_{\alpha, \beta \in I}$, где $\sigma_{\alpha \beta}=\sigma_{\beta}-\sigma_{\alpha}$ есть 1-коцикл покрытия $\left\{\mathscr{U}_{\alpha}\right\}$ со значениями в пучке $\underline{\mathbf{J}}$ голоморфных (либо регулярных) сечений $\mathbf{J}$, а $\mathfrak{M}$ естественно отождествляется с открытым подмножеством торсора $\mathbf{J}^{c}$, построенного по $c$. Следующая лемма суммирует некоторые простые факты о соотношении между структурами лагранжевых расслоений на абелевой схеме и ее торсорах.

Лемма 5.4. Пусть $B$ - неособое многообразие размерности $n$ u $f: A \rightarrow$ $B$ - гладкое семейство связных коммутативных алгебраических групп (либо комплексных групп Ли) такие, что общий слой $A_{b}$ есть абелево многообразие (либо компактный комплексный тор) размерности п. Пусть с-произвольный класс когомологий из $H^{1}(B, \underline{A})$ в әтальной (либо классической) топологии и $g: X=A^{c} \rightarrow B$ - торсор, построенный по с. Тогда выполнены следующие утверждения.

\footnotetext{
${ }^{3}$ Ясно, что форма $\Lambda_{\mathscr{U}, s}$ зависит от выбора сечения $s: \mathscr{U} \rightarrow \mathscr{H}$.
} 
(i) Пусть Х обладает квазисимплектической структурой $\Lambda_{X}$ такой, что g есть лагранжево расслоение по отношению $\kappa \Lambda_{X}$. Тогда $A$ также обладает квазисимплектической структурой $\Lambda_{A}$, для которой $f$ является лагранжевым расслоением, и для любого локального сечения $s: V \rightarrow X$ проекции $g$, индуцирующего изоморфизм $i_{s}: X_{V}=g^{-1}(V) \stackrel{\sim}{\rightarrow} A_{V}=f^{-1}(V)$, существует 2-форма $\omega_{V} \in H^{0}\left(V, \Omega^{2}\right)$ такая, что $\Lambda_{X}-i_{s}^{*} \Lambda_{A}=g^{*} \omega_{V}$ на V. Будем говорить, что такие квазисимплектические структуры $\Lambda_{X}, \Lambda_{A}$ ассочиированы друг другу.

(ii) Если $\Lambda_{X}, \Lambda_{A}-$ ассочиированнье квазисимплектические структуры, mо любая пара $\Lambda_{1, X} \in \Lambda_{X}+g^{*} H^{0}\left(B, \Omega^{2}\right), \quad \Lambda_{1, A} \in \Lambda_{A}+f^{*} H^{0}\left(B, \Omega^{2}\right)$ есть также пара ассочиированных квазисимплектических структур. Более того, всегда существует такая структура $\Lambda_{1, A}$, которая является симплектической.

В частности, если $H^{0}\left(B, \Omega^{2}\right)=0$ и если существует пара $\Lambda_{X}, \Lambda_{A}$ ассочиированных квазисимплектических структур, то она единственна, и $\Lambda_{A}$ на самом деле является симплектической.

Структура $\Lambda_{A}$, ассочиированная с данной структурой $\Lambda_{X}$, также единственна и симплектична, если мы наложим дополнительное условие, что ограничение $\Lambda_{A}$ на сечение из нейтральных әлементов слоев проекции $f$ тождественно равно нулю.

(iii) Обратно, пусть А обладает квазисимплектической структурой $\Lambda_{A}$ такой, что $f$ есть лагранжево расслоение по отношению $\kappa \Lambda_{A}$. Пусть также выполнено по крайней мере одно из двух условий: $H^{1}\left(B, \Omega^{2}\right)=0$ или с имеет конечный порядок. Тогда $X$ обладает квазисимплектической структурой $\Lambda_{X}$, ассоциированной $с \Lambda_{A}$. В случае, когда с имеет конечный порядок, можно выбрать симплектическую структуру $\Lambda_{X}$.

(iv) В алгебрачческой категории $H_{\mathrm{ett}}^{1}(B, \underline{A})$ является группой кручения и любой торсор с квазисимплектической лагранжевой структурой также имеет симплектическую структуру.

ДокАЗАТЕЛЬство. Для доказательства утверждений (i), (ii) см. предложения $2.3,2.6$ в [10]. В самом деле, условия этих предложений сформулированы там для симплектических структур, но доказательства остаются верными также для квазисимплектических структур. Предложение 2.6 из [10] рассматривает случай утверждения (iii), когда $H^{1}\left(B, \Omega^{2}\right)=0$. Нам необходимо ввести некоторые пункты этого доказательства для объяснения случая, когда $c$ имеет конечньй порядок.

Для любого $b \in B$ и для достаточно малой окрестности $V$ точки $b$ существуют локальные параметры $u_{1}, \ldots, u_{n}$ на $V$ и локальное сечение $s_{V}$ проекции $g_{V}: X_{V} \rightarrow V$, отождествляющее $X_{V}$ с $A_{V}$ таким образом, что $s_{V}$ становится сечением $e$ из нейтральных элементов слоев проекции $f$. Можно реализовать $A_{V}$ как фактор $\Omega_{V}^{1} / \mathscr{L}$ кокасательного расслоения к $V$ по локальной системе $\mathscr{L}$ ранга $2 n$ в обшей точке. Пусть $z_{1}, \ldots, z_{n}$ - координаты в $\Omega_{B}^{1}$, двойственные к $d u_{1}, \ldots, d u_{n}$. Тогда ограничение структуры $\Lambda_{A}$ на $A_{V}$ может быть записано в форме

$$
\Lambda_{X}=\sum_{p} d u_{p} \wedge d z_{p}+\beta, \quad \beta=\sum_{p, q} \beta_{p q}(u) d u_{p} \wedge d u_{q}
$$


Здесь $\beta$ не зависит от $z$, как было доказано в [10]. Пусть $\Lambda_{0, A}=\sum_{p} d u_{p} \wedge d z_{p}=$ $\Lambda_{X}-\beta$. Инвариантность $\Lambda_{0, A}$ относительно сдвигов на сечения из $\mathscr{L}$ эквивалентна равенству

$$
d\left(\sum_{p} \gamma_{p} d u_{p}\right)=0
$$

где $\gamma=\left(\gamma_{1}, \ldots, \gamma_{n}\right)$ - любое локальное сечение пучка $\mathscr{L}$. Следовательно, мы имеем также инвариантность относительно сдвигов на сечения из $\mathbb{Q} \otimes \mathscr{L}$, откуда следует случай конечного порядка.

Утверждение (iv) получается последовательным применением утверждений (i) и (iii).

Теперь можно доказать следуюшую теорему.

Теорема 5.5. Существует симплектическая структура $\tilde{\wedge}_{\mathfrak{M}}$ на $\mathfrak{M}$ такая, что выполнены следующие два условия:

(a) $\tilde{\Lambda}_{\mathfrak{M}}-\wedge_{\mathfrak{M}} \in H^{0}\left(U, p^{*} \Omega_{U}^{2}\right)$, следовательно, р есть лагранжево расслоение относительно $\tilde{\wedge}_{\mathfrak{M}}$, и $\tilde{\Lambda}_{\mathfrak{M}}$ также удовлетворяет заключению теоремы 5.2;

(b) $\tilde{\Lambda}_{\mathfrak{M}}$ продолэсается до симплектической структуры $\Lambda_{\mathbf{J}^{c}}$, ассочиированной $c \Lambda_{\mathbf{J}}$.

ДокАЗАТЕЛЬСТво. Мынаходимся в алгебраической ситуации, поэтому можно применить лемму 5.7 , (iii) для построения симплектической структуры $\Lambda_{\mathbf{J}} c$, ассоциированной с формой Донаги-Маркмана $\Lambda_{\mathbf{J}}$. Положим $\tilde{\Lambda}_{\mathfrak{M}}=\left.\Lambda_{\mathbf{J}}\right|_{\mathfrak{M}}$, так что выполнено условие (b). Определение отношения ассоциированности в лемме 5.4, (i) и теорема 5.2 также дают утверждение (a).

ЗАмечАниЕ 5.6. Мы высказываем гипотезу, что $\wedge_{\mathfrak{M}}$ является симплектической структурой.

ЗАмЕЧАНИЕ 5.7. Класс этальных когомологий $c$, определяюший $\mathbf{J}^{c}$, имеет порядок 3 , поскольку кривая $3 C$ может быть отображена в $J^{2}(X)$, где $C \subset X=Y \cap H$ есть нормальная эллиптическая квинтика, каноническим образом, используя пятикратное плоское сечение кубики $Y$ в качестве кривой отсчета для определения отображения Абеля-Якоби. Поэтому, кроме тривиального торсора $\mathbf{J}$ и торсора $\mathbf{J}^{c}$, существует еше один более интересный торсор $\mathbf{J}^{c^{2}}$. Какова геометрическая интерпретация этого торсора?

ЗАмЕчАниЕ 5.8. Можно продолжить нашу конструкцию на большее открытое множество $\bar{U}$ такое, что $\operatorname{codim} \check{\mathbb{P}}^{5} \backslash \bar{U} \geqslant 2$. Получим те же теоремы $5.2,5.5$ для (квази)симплектических структур $\Lambda_{\overline{\mathbf{J}}}, \Lambda_{\overline{\mathbf{J}^{c}}}, \Lambda_{\overline{\mathfrak{M}}}$. Поскольку $H^{0}\left(\bar{U}, \Omega^{2}\right)=0$, ассоциированные квазисимплектические структуры на продолженных семействах $\bar{h}: \overline{\mathbf{J}} \rightarrow \bar{U}, \bar{h}^{c}: \overline{\mathbf{J}}^{c} \rightarrow \bar{U}$ единственны и симплектичны.

Рассмотрим в качестве $\bar{U}$ множество гиперплоскостей $H$ таких, что $H \cap Y$ имеет не более одной невырожденной двойной точки в качестве особенности. Любая кубика $X$, имеюшая лишь одну изолированную особую точку, содержит нормальную эллиптическую квинтику, не проходящую через особую точку. Чтобы это увидеть, возьмем гиперплоское сечение кубики $X$, являющееся гладкой кубической поверхностью $S \subset \mathbb{P}^{3}$. Оно содержит 5-мерную линейную систему квинтик 
арифметического рода 1 , и ее общий член есть неособая пространственная эллиптическая кривая $C$. По лемме 2.7, с) из [11], доказательство которой использует лишш гладкость $X$ в точках поверхности $S$, имеем $h^{0}\left(\mathscr{N}_{C / X}\right)=10, h^{1}\left(\mathscr{N}_{C / X}\right)=0$. Следовательно, схема Гильберта кубики $X$ неособа размерности 10 в точке $[C]$. Семейство квинтик рода 1 в гиперплоских сечениях кубики $X$ 9-мерно, так что видно, что $C$ деформируется в кривую, не лежашую в 3 -мерном пространстве, которая и есть желаемая нормальная эллиптическая квинтика.

Определим теперь $M_{X}$ как подмножество в пространстве модулей пучков на $X$, состояшее из векторных расслоений ранга 2, полученных конструкцией Серра из всех нормальных эллиптических квинтик на $X$, не проходящих через особую точку кубики $X$, и пусть $\overline{\mathfrak{M}}$ есть объединение множеств $M_{X}$ по всем $X=Y \cap H$ с $H \in$ $\bar{U}$. Все наши доказательства, касающиеся размерности и неособости $M_{X}, \mathfrak{M}$, равно как и определение и доказательство невырожденности формы $\Lambda_{\mathfrak{M}}$, остаются справедливыми после замены $\mathfrak{M}$ на $\overline{\mathfrak{M}}$ для всех $H \in \bar{U}$. Поэтому мы получаем квазисимплектическую структуру Ионеды $\Lambda_{\overline{\mathfrak{M}}}$ на $\overline{\mathfrak{M}}$.

Донаги и Маркман [5, пример 8.22] замечают, что их симплектическая структура продолжается до 2-формы $\bar{\Lambda}_{\overline{\mathbf{J}}}$ на обобщенном относительном промежуточном якобиане $\bar{h}: \overline{\mathbf{J}} \rightarrow \bar{U}$. Они указывают, что нетрудно видеть, что она не вырождена на гранище. Это также легко следует из нашего рассмотрения.

В самом деле, невырожденность формы $\Lambda_{\overline{\mathfrak{M}}}$ влечет, что вертикальное касательное расслоение для $\overline{\mathfrak{M}}$ изоморфно $\Omega \frac{1}{U}$, следовательно, ассоциированная групповая схема $\overline{\mathbf{J}}$ изоморфина $\Omega \frac{1}{U} / \mathscr{L}$, как и в доказательстве леммы 5.4. Стандартная симплектическая структура кокасательного расслоения к $\bar{U}$ спускается на фактор по $\mathscr{L}$ над $U$, а значит, также и над $\bar{U}$, поскольку условие спуска $(5.21)$ продолжается по непрерывности. Таким образом, мы получаем симплектическую структуpy $\Lambda_{\overline{\mathbf{J}}}$ на $\overline{\mathbf{J}}$. Так как ограничения форм $\Lambda_{\overline{\mathbf{J}}}, \bar{\Lambda}_{\overline{\mathbf{J}}}$ на $U$ ассоциированы с одной и той же квазисимплектической структурой на $\mathbf{J}^{c}$, то они отличаются на поднятую 2-форму с $U$. Следовательно, они определяют один и тот же изоморфизм между $\Omega_{\bar{U}}^{\frac{1}{U}} \mathscr{T}_{\overline{\mathbf{J}} / \bar{U}}$ над $U$, который является тождественным для $\Lambda_{\overline{\mathbf{J}}}$. По непрерывности это выполняется также над $\bar{U}$. Следовательно, $\bar{\Lambda}_{\overline{\mathbf{J}}}$ невырождена. Поскольку $H^{0}\left(\bar{U}, \Omega^{2}\right)=0$, мы имеем единственность над $\bar{U}$, следовательно, $\bar{\Lambda}_{\overline{\mathbf{J}}}=\Lambda_{\overline{\mathbf{J}}}$.

Продолжение теоремы 5.2 на $U$ вытекает из следуюшего наблюдения: если регулярное сечение $\Lambda_{\overline{\mathfrak{M}}}-\Psi_{\mathscr{U}}^{*} \Lambda_{\overline{\mathbf{J}}}$ векторного расслоения $\Omega_{\frac{2}{\mathfrak{M}}}^{2}$ над $\mathscr{U}$ принадлежит векторному подрасслоению $\bar{p}^{*} \Omega_{\bar{U}}^{2}$ над $U \cap \mathscr{U}$, то оно принадлежит ему над всем $\mathscr{U}$.

\section{Список литературы}

1. Beauville A. Variétés Kaehleriennes dont la première classe de Chern est nulle // J. Differ. Geom. 1983. V. 18. P. 755-782.

2. Beauville A., Donagi $R$. La variété des droites d'une hypersurface cubique de dimension 4 // C. R. Acad. Sci. Paris. Ser. I. Math. 1985. V. 301. № 14. P. 703-706.

3. Bottacin F. Poisson structures on moduli spaces of sheaves over Poisson surfaces // Invent. Math. 1995. V. 121. P. 421-436.

4. Clemens C. H., Griffiths P. A. The intermediate Jacobian of the cubic threefold // Annals of Math. 1972. V. 95. P. 281-356.

5. Donagi R., Markman E. Spectral covers, algebraically completely integrable Hamiltonian systems, and moduli of bundles // Integrable systems and quantum groups. CIME Lectures. 
Italy, June 14-22, 1993 / Ed. M. Francaviglia. Berlin: Springer-Verlag, 1996. P. 1-119.

6. Hulek K. Projective geometry of elliptic curves // Astérisque. 1986. V. 137.

7. Huybrechts D., Lehn M. The geometry of moduli spaces of sheaves. Aspects of Mathematics. E31. Braunschweig: Vieweg, 1997.

8. Iliev A., Markushevich D. The Abel-Jacobi map for a cubic threefold and periods of Fano threefolds of degree 14 // Doc. Math. 2000. V. 5. P. 23-47.

9. Kobayashi S. Simple vector bundles over symplectic Kaehler manifolds // Proc. Japan Acad. Ser. A. 1986. V. 62. P. 21-24.

10. Markushevich D. Lagrangian families of Jacobians of genus 2 curves // J. Math. Sciences. 1996. V. 82. № 1. P. 3268-3284.

11. Markushevich D., Tikhomirov A.S. The Abel-Jacobi map of a moduli component of vector bundles on the cubic threefold // J. Alg. Geom. 2001. V. 10. P. 37-62.

12. Mukai S. Symplectic structure of the moduli of sheaves on an abelian or K3 surface // Invent. Math. 1984. V. 77. P. 101-116.

13. O'Grady K. G. Algebro-geometric analogues of Donaldson's polynomials // Invent. Math. 1992. V. 107. P. 351-395.

14. Simpson C. T. Moduli of representations of the fundamental group of a smooth projective variety. I // Publ. Math. I. H.E.S. 1994. V. 79. P. 47-129.

15. Тюрин A.H. Симплектические структуры на многообразиях модулей векторных рассмотрений на поверхностях $p_{g}>0 / /$ Изв. АН СССР. Сер. матем. 1988. Т. 52. № 4 . C. $813-852$.

16. Voisin $C$. Sur la stabilité des sous-variétés lagrangiennes des variétés symplectiques holomorphes // Complex Projective Geometry. Cambridge: Cambridge Univ. Press, 1992. P. 294-303.

17. Welters G. E. Abel-Jacobi isogenies for certain types of Fano threefolds // Mathematical Centre Tracts. V. 141. Amsterdam, 1981.

Ярославский государственный педагогический университет

E-mail: tikhomir@yaroslavl.ru

Поступило в редакцию

10.IX.2001 